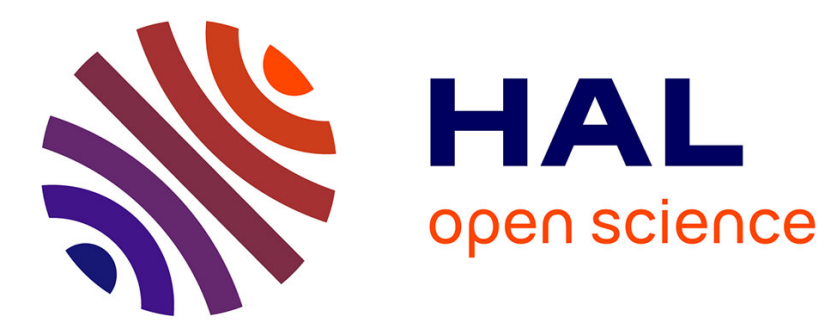

\title{
High-accurate and robust conservative remapping combining polynomial and hyperbolic tangent reconstructions
}

\author{
Milan Kucharik, Raphaël Loubère
}

\section{To cite this version:}

Milan Kucharik, Raphaël Loubère. High-accurate and robust conservative remapping combining polynomial and hyperbolic tangent reconstructions. Computers and Fluids, 2020, 208, pp.104614. 10.1016/j.compfluid.2020.104614 . hal-03084461

\section{HAL Id: hal-03084461 \\ https://hal.science/hal-03084461}

Submitted on 22 Dec 2020

HAL is a multi-disciplinary open access archive for the deposit and dissemination of scientific research documents, whether they are published or not. The documents may come from teaching and research institutions in France or abroad, or from public or private research centers.
L'archive ouverte pluridisciplinaire HAL, est destinée au dépôt et à la diffusion de documents scientifiques de niveau recherche, publiés ou non, émanant des établissements d'enseignement et de recherche français ou étrangers, des laboratoires publics ou privés. 


\title{
High-accurate and robust conservative remapping combining polynomial and hyperbolic tangent reconstructions
}

\author{
Milan Kucharik ${ }^{\mathrm{b}}$, Raphaël Loubère ${ }^{\mathrm{a}}$ \\ ${ }^{a}$ Institut de Mathématiques de Bordeaux (IMB), Université de Bordeaux, CNRS, Bordeaux INP, F33400, Talence, France \\ ${ }^{b}$ Faculty of Nuclear Sciences and Physical Engineering, Czech Technical University in Prague, Brehova 7, Praha 1, Czech Republic
}

\begin{abstract}
In this article we present a 1D single-material conservative remapping method that relies on high accurate reconstructions: polynomial $\left(\mathbb{P}_{4}, \mathbb{P}_{1}\right.$ with slope limiter) and non-linear hyperbolic tangent (THINC) representations. Such remapping procedure is intended to be used pairwise with a cell-centered Lagrangian scheme along with a rezone strategy to build a so-called indirect Arbitrary-Lagrangian-Eulerian scheme. Most of practically used Lagrangian schemes are second-order accurate. The goal of this work is to handle with accuracy contact using THINC reconstructions. At the same time, the smooth part of the solution is dealt with quartic polynomials, resulting locally in fifth order accurate remapping method. To ensure robustness, TVD-like reconstructions $\left(\mathbb{P}_{1}\right.$ with slope limiter) are employed otherwise. A simple feature tracking algorithm is designed to assign a reconstruction type per cell $\left(\mathbb{P}_{4}, \mathbb{P}_{1}^{\text {lim }}\right.$ or THINC). This tracking algorithm is based on the nature of the contact waves which are traveling at the fluid velocity, while the shocks are compressive and detectable by following a change of cell volumes. Numerical results assess the behavior of such a remapping method on pure remapping problems of a scalar quantity and in the context of the full hydrodynamics equations. The associated indirect cell-centered ALE numerical scheme is run and produces numerical results that are presented to assess the extreme accuracy gained by such a remapping procedure employing a mix of reconstruction types.
\end{abstract}

Key words: Remapping, polynomial reconstruction, high accuracy, indirect ALE, interface tracking THINC reconstruction, hydrodynamics

\section{Contents}

1 Introduction $\quad 2$

2 Abstract high accurate remapping scheme $\quad 3$

3 Cell-centered Lagrangian scheme to solve the hydrodynamics system of conservation laws 6

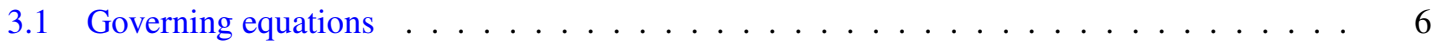

3.2 Lagrangian scheme . . . . . . . . . . . . . . . . . . . . . 7

3.2.1 First order discretization . . . . . . . . . . . . . . . . . . . 7

3.2 .2 Second order discretization . . . . . . . . . . . . . . . . . 8

Email addresses: kucharik@newton.fjfi.cvut.cz (Milan Kucharik), raphael.loubere@u-bordeaux.fr (Raphaël Loubère) 
4 High-accurate indirect ALE scheme $\quad 9$

4.1 Classical second-order remap scheme: piece-wise linear reconstruction + slope limiter . . 10

4.2 High accurate remapping scheme . . . . . . . . . . . . . . . . . . . . . . 10

4.2.1 High accuracy $\mathbb{P}_{4}$ : Quartic polynomial reconstruction . . . . . . . . . . . . 11

4.2.2 Non-oscillatory $\mathbb{P}_{1}^{\lim }$ : Piece-wise linear polynomial reconstruction with slope limiter 11

4.2.3 Interface sharpening THINC : Non-linear hyperbolic tangent reconstruction . . . 11

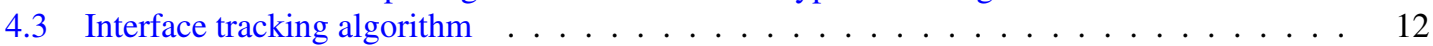

4.4 Summary of the indirect ALE scheme . . . . . . . . . . . . . . . . . . . 13

5 Numerical experiments for static reconstruction and remap 14

5.1 Static reconstruction . . . . . . . . . . . . . . . . . . . . . . 14

5.2 Static remap . . . . . . . . . . . . . . . . . . . . . . 14

6 Numerical experiments for the full hydrodynamics equations 17

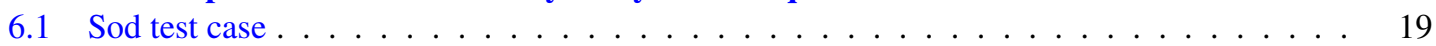

6.2 Lax test case . . . . . . . . . . . . . . . . . . . . . . . . . . . . . . . . . . . . . . .

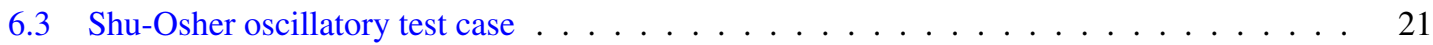

6.4 Woodward-Collela blastwave . . . . . . . . . . . . . . . . . . 22

7 Conclusions and perspectives 29

\section{Introduction}

The origins of Lagrangian numerical schemes are probably to be found in classified documents from the Los Alamos National Laboratory, in New Mexico state in the U.S.A, during World War II and the Manhattan project where the "calculation of certain time-dependent fluid flows played an important part in the wartime work of the laboratory" (preface to the first edition of [1]). Indeed, the Lagrangian formulation of the equations of hydrodynamics has a very old and venerable history. The very first numerical calculations that resemble modern computer simulations in the numerical issues considered fluid equations in the Lagrangian frame of reference in 1D [2]. Starting at this epoch, the staggered Lagrangian numerical scheme using artificial viscosity term became popular and deeply analyzed, see for instance $[3,4,5,6,7]$ and the recent review [8]. An alternative to the previous staggered discretizations is to derive a Lagrangian scheme based on the cell centered Godunov method [9]. A major advance in this direction has been made in [10, 11], proving viability of cell centered schemes for a vast range of applications, see also [8]. In recent years, the field of cell centered schemes is under a very active research and many groups contributed to this topic, see for example[12, 13, 14, 15, 16, 17] for a representative set of examples.

Usually, to extend the robustness and usability of a Lagrangian scheme, it is supplemented with a rezone/remesh procedure and a conservative remap algorithm to produce a so-called indirect ArbitraryLagrangian-Eulerian method [18, 19, 20, 21, 22, 23, 24]. This technique prevents mesh degenerations in regions of shear flows by consecutive improvement of the computational mesh during the rezone phase. A critical part of the indirect ALE algorithm is the remap phase transferring fluid state quantities from the Lagrangian to the rezoned mesh, which we focus on in this paper. Providing an exhaustive list of contributors to this field is almost impossible, as this should embrace the key words interpolation techniques, advection methods, (flux-corrected) transport methods, and remapping in general. However, some very much related works are to be found in $[19,25,26,27,28,29,30]$. The readers are urged to refer to the review made by Benson [19] to have an overview of legacy remapping methods. At first glance, defining a conservative remapping technique between an old and a new mesh for a single constant variable (say mass), constant in each cell, seems trivial. First, the exact geometrical intersections between a new cell and the old mesh are computed. Second, the new mass in the new cell is computed as the sum of all old masses present in the intersection sub-cells. This straightforward method is unfortunately terribly inaccurate and overly 
diffuses the quantity profile. This has led several authors to reconstruct the underlying function as a piecewise linear function [26, 27]. As such, the remapping method is exact for a global linear function and is considered as second-order accurate. However, the generation of non-physical remapped quantities enforces the utilization of slope or flux limiters during the reconstruction step. Such slope limiters may not always be trivial to design even if there exists a vast literature about slope/flux limiters, some of them are now known after their discoverers (van Leer [31, 32, 33], van Albada [34], Sweby [35], Barth-Jespersen [36], Venkatakrishnan [37], Koren [38, 39], etc.) or their particularity (minmod [40], superbee [40], monotonized central [32], etc.). Relatively few attempts have been made to increase the remapping accuracy to more than second order via high order polynomial reconstructions, for several examples see [41, 42, 43]. Any attempt has to face the difficulty of designing a 'limiter' for non-smooth solution. And the notion of limiting becomes obscur if not contrived for higher order polynomial reconstructions.

Usually, the use of polynomial based reconstruction is rarely questioned even if any type of function could be employed instead. In the context of designing high accurate Finite Volume schemes some authors recently associate hyperbolic tangent (THINC) and polynomials as reconstruction basis, see for instance [44, 45, 46, 47, 48, 49]. Moreover, in the remapping context, the mechanism used to handle discontinuous solution (contact, shock) systematically adds some artificial numerical dissipation, leading the spreading of interfaces over several cells: two for shocks, five or more for contacts.

In this work we propose to adapt the reconstruction procedure for instance by using high accurate polynomials in smooth areas, non-linear hyperbolic tangent function for contact, and limited piece-wise linear polynomials on irregular area. Then, we have to design an algorithm to detect such zones and have a high-quality representation of the quantity over various features in the flow.

This paper is a 1D proof of concept: it is intended to design an indirect single-material cell-centered ALE scheme for which the remapping is able to maintain extremely sharp contact discontinuities, an accurate solution in smooth regions, and an essentially non-oscillatory one close to shocks and steep gradients. We will design the simplest possible tools in order to show the capability of such an approach and illustrate the gain brought by the use of high accurate polynomial and non-linear reconstructions in the hydrodynamics context.

The paper is organized as follows. Section 2 will present the basics of classical high accurate remapping techniques as a conservative interpolation procedure. In section 3, we present the cell-centered Lagrangian scheme to be coupled with the remap to form an indirect ALE scheme. Then in section 4, we propose particular improvements based on extending the basis of reconstruction functions with high accurate polynomial and non-polynomial functions. A procedure intended to track contact and follow shock area is presented in subsection 4.3, and its operational transplant into a 1D indirect ALE code is described in subsection 4.4. The following section 5 is devoted to present the numerical results obtained on classical static tests, while full hydrodynamics test cases are addressed in section 6 . A conclusion and perspective section 7 closes this paper.

\section{Abstract high accurate remapping scheme}

In order to set up the notation, let us describe the remapping environment for a generic function $u$ defined on domain $\Omega$. The computational domain is paved with an original mesh $O$ made of $I$ cells, not necessarily of uniform size $\Delta x_{i}, i=1, \ldots, I$ and denoted $I_{i}=\left[x_{i+1 / 2}, x_{i-1 / 2}\right]$ :

$$
O=\left\{I_{i}=\left[x_{i+1 / 2}, x_{i-1 / 2}\right], \quad i=1, \ldots, I\right\} .
$$

The cell center is denoted $x_{i}=\frac{1}{2}\left(x_{i+1 / 2}+x_{i-1 / 2}\right)$. The space variable in $O$ is denoted $x$ and we assume that an integrable function $\varphi^{e x}(x)$ is defined on this domain. Moreover, we assume that the function $\varphi^{e x}$ is characterized by its mean values over each cell $I_{i}$ as

$$
\varphi_{i}=\frac{1}{\Delta x_{i}} \int_{I_{i}} \varphi^{e x}(x) d x .
$$


The vector of all mean values is called the state vector and denoted by $\varphi_{O}=\left(\varphi_{1}, \ldots, \varphi_{M}\right)$. The original mesh $O$ and the state vector $\varphi_{O}=\left(\varphi_{1}, \ldots, \varphi_{M}\right)$ emulate the resulting solution after a Lagrangian scheme has operated.

Next, a target mesh $\mathcal{T}$ made of $J$ non-uniform cells called $J_{j}, j=1, \ldots, J$, is also considered:

$$
\mathcal{T}=\left\{J_{j}=\left[x_{j+1 / 2}, x_{j-1 / 2}\right], \quad j=1, \ldots, J\right\} .
$$

The number of cells $I$ may be different than $J$ and the location of cells $J_{j}$ may not have any relation with those of cells $I_{i}$, meaning that the old and target mesh may not have any logical relation between each other apart from the fact that both are paving $\Omega$. The target mesh can be though as the mesh produced by the rezone strategy of an indirect ALE scheme. Note that the super-mesh (or overlay or exact intersection) of $O$ and $\mathcal{T}$, called $\mathcal{I}$, is constituted by the intersections of cells

$$
K_{i, j}=I_{i} \bigcap J_{j}, \quad i=1, \ldots, I, \quad j=1, \ldots, J
$$

where $K_{i, j}$ is empty if $I_{i}$ and $J_{j}$ do not intersect. Then if one orders the non-empty intersection cells $K_{i, j}$ and enumerates them by a single index from $k=1$ to $K$, then we can define the intersection mesh as

$$
\mathcal{I}=\left\{K_{k}=\left[x_{k+1 / 2}, x_{k-1 / 2}\right], \quad k=1, \ldots, K\right\},
$$

$K_{k}$ being an intersection cell. We can remark that $x_{k+1 / 2}$ and $x_{k-1 / 2}$ coincide with either one $x_{i+1 / 2}, x_{i-1 / 2}$ or $x_{j+1 / 2}, x_{j-1 / 2}$. It is also useful to remark that by construction we have

$$
I_{i}=\bigcup_{j=1}^{J} I_{i} \cap J_{j}=\bigcup_{k: K_{k} \in I_{i}} K_{k} \text {, and } J_{j}=\bigcup_{i=1}^{I} I_{i} \cap J_{j}=\bigcup_{k: K_{k} \in J_{j}} K_{k} \text {, }
$$

so every initial and target cell can be composed from the intersection cells $K_{k}$.

The purpose of a conservative remapping scheme consists in transferring the information hold by $\varphi_{O}$ from the original mesh onto the target one. In an indirect ALE context, the remap transfers the state vector from the Lagrangian mesh onto the rezoned one. In other words, we wish to compute the integral mean value

$$
\varphi_{j}=\frac{1}{\Delta x_{j}} \int_{J_{j}} \varphi^{e x}(x) d x
$$

only knowing the mean values $\varphi_{i}$ defined on the original mesh. A simple manipulation using (4) and (6) yields

$$
\varphi_{j}=\frac{1}{\Delta x_{j}} \int_{J_{j}} \varphi^{e x}(x) d x=\frac{1}{\Delta x_{j}} \sum_{i=1}^{I} \int_{K_{i, j}=I_{i} \cap J_{j}} \varphi^{e x}(x) d x,
$$

which would produce the exact mean value of $\varphi^{e x}$ over target cell $J_{j}$ if one would know the exact function $\varphi^{e x}$ over cell $I_{i}$. Unfortunately we have only access to the mean values in a neighborhood $\left(\varphi_{i-h}, \ldots, \varphi_{i+h}\right)$ with $h>0$ to be specified.

In order to design a high accurate remapping scheme, one first needs to design an accurate representation of the unknown function $\varphi^{e x}$ only by a clever use of its mean values $\varphi_{O}$. This step is called 'reconstruction', and it is denoted in cell $I_{i}$ as: $\widehat{\varphi}_{i}(x) \equiv \mathrm{R}_{i}(x)$, where the reconstruction operator $\mathrm{R}$ can be written in an abstract form for all $x$ in cell $I_{i}$ as

$$
\mathrm{R}:\left(x, I_{i}, \mathcal{S}_{i}^{p, q}\right)_{4} \longrightarrow \widehat{\varphi}_{i}(x)
$$


where $\mathcal{S}_{i}^{p, q}$ is the stencil associated to cell $I_{i}$ made of $p$ cells to the left and $q$ cells to the right, that is $\mathcal{S}_{i}^{p, q}=\left(\varphi_{i-p}, \ldots, \varphi_{i+q}\right)$. Note that $p$ and/or $q$ could be equal to zero for instance if shifted stencils are to be employed. The operator $\mathrm{R}$ is usually working in the simplest space, that is the approximate function space is the space of polynomials. Even simpler, classical reconstruction procedures only consider piece-wise polynomial representation of degree $d=1$ or $d=2$ in cell $I_{i}$ given $p=q=1$. However, in this work we also use $d=4$ polynomial degree with central stencil $p=q=2$. The choice of the approximate function space is referred to as the 'representation space'. In other words, the exact function $\varphi^{\text {ex }}(x)$ is approximated in cell $I_{i}$ by $\widehat{\varphi}_{i}(x)$, which belongs to a given set of possible representation functions: polynomials $\mathbb{P}_{1}, \mathbb{P}_{4}$ or exponential, trigonometric functions, etc.

The remap procedure then consists in replacing the exact function $\varphi^{e x}(x)$ in (8) by its approximation $\widehat{\varphi}_{i}(x)$ in cell $I_{i}$ as

$$
\varphi_{j}=\frac{1}{\Delta x_{j}} \sum_{i=1}^{I} \int_{I_{i} \cap J_{j}} \widehat{\varphi}_{i}(x) d x
$$

If the reconstructions are more complicated functions, the integrals in the previous equation are approximated by a $G$ point quadrature rule to get

$$
\varphi_{j}=\frac{1}{\Delta x_{j}} \sum_{i=1}^{I} \int_{I_{i} \cap J_{j}} \widehat{\varphi}_{i}(x) d x \simeq \frac{1}{\Delta x_{j}} \sum_{i=1}^{I} \sum_{g=1}^{G} w_{g} \widehat{\varphi}_{i}\left(x_{g}\right),
$$

where $\left(w_{g}, x_{g}\right)$ are the quadrature weight and the position respectively. In summary, given a target cell $J_{j}$, one first identifies the cell $I_{i}$ such that $K_{i, j} \neq \emptyset$. Then the reconstruction operator acts on those cells $I_{i}$ to get the representation/reconstruction: $\widehat{\varphi}_{i}$. Last, the integration rule is applied to get the final mean value $\varphi_{j}$ using (11).

Some expected properties of a conservative transfer from the old onto the target mesh are:

1. a local conservation: the mean value represented by $\varphi_{i}$ in cell $I_{i}$ should be scattered in its vicinity,

2. a high accuracy on the smooth parts of the flow,

3. the robustness, and an essentially non-oscillatory behavior, possibly accurate description of discontinuities.

While the first two properties are relatively simple to assure, the last one is more difficult. Indeed the robustness is usually ensured, but the accurate description of discontinuities is often replaced by an excessive dissipation to avoid spurious oscillations.

The accuracy of the previous formula is almost entirely linked to the reconstruction procedure $\mathrm{R}_{i}$, and, possibly the accuracy of the integral rule. In $1 \mathrm{D}$, there is no mesh intersection error. Moreover, the integration rule is often, if not always, chosen of high enough accuracy so that the resulting error remains at a lower level compared to the other errors. Finally, only the representation and reconstruction errors remain. The representation error is linked to the choice of functions employed to mimic the underlying (unknown) exact solution, whereas the reconstruction error is linked to the ability to choose the best approximate function in the representation function space. For instance, the representation space constituted by the high degree $d$ polynomials is appropriate when the underlying solution is smooth enough and there are good candidate functions in this space to lower the reconstruction error. However, if $\varphi^{e x}$ is discontinuous then there is no good polynomial in this space to cope with the situation, hence leading to a large reconstruction error due to the fact that the representation space is inappropriate. Therefore the reconstruction error over the mesh is the sum of the cell reconstruction error made by the specific choice of one of the representative function. For instance, classical remapping procedures [50, 51, 27, 26, 29, 52] usually employ piece-wise linear polynomial functions as representation basis. Some more advanced ones use high-order polynomials [41, 42, 43]. Even if polynomial basis is the simplest possibility, any choice of representation basis is possible and in this work, we consider the polynomials and hyperbolic tangent representation space. 


\section{Cell-centered Lagrangian scheme to solve the hydrodynamics system of conservation laws}

In the Lagrangian hydrodynamics methods, a computational cell moves with the flow velocity. This ensures that there is no mass flux crossing the boundary of the Lagrangian moving cell. Thus, the Lagrangian methods can capture contact discontinuity sharply in multi-material fluid flows. However, in the Lagrangian framework, one has to discretize not only the gas dynamics equations but also the vertex motion in order to move the mesh. The most natural way to solve this problem employs a staggered discretization in which position, velocity and kinetic energy are centered at points, while density, pressure and internal energy are within cells. The dissipation of kinetic energy into internal energy through shock waves is ensured by an artificial viscosity term. Since the seminal works of von Neumann and Richtmyer [3], and Wilkins [4], many developments have been made in order to improve the accuracy and the robustness of staggered hydrodynamics $[53,54,7,55]$. More specifically, the construction of a compatible staggered discretization leads to a scheme that conserves total energy in a rigorous manner [6].

An alternative to the staggered discretization is to derive a Lagrangian scheme based on the Godunov method $[9,10,11,56,57,58]$. In comparison to staggered discretizations, Godunov-type methods exhibit the good property of being naturally conservative, they do not need an artificial viscosity. In the Godunov-type method approach, all conserved quantities, including momentum, and hence cell velocity are cell-centered. Consequently, they allow a straightforward implementation of conservative remapping methods when they are used in the context of an Arbitrary-Lagrangian-Eulerian (ALE) strategy.

In this section we focus on the development of a cell-centered Lagrangian scheme to be used as the engine of a 1D hydrodynamics ALE code. First, we present the governing hydrodynamics equations in a Lagrangian formalism. Then, the cell-centered Lagrangian scheme from Maire [11, 59, 56, 58] is presented in its first and second order accurate versions, see also the reviews [8,24]. A very interesting presentation of the derivation of Lagrangian equations and schemes can be found in [58 $]^{1}$.

\subsection{Governing equations}

Let us consider a fluid particle initially located at position $X$ and denote its position at time $t$ by $x(X, t)$. Here, $X$ denotes the Lagrangian coordinate. If $u=u(x, t)$ denotes the $x$-component of the fluid velocity, then the fluid paths are described by the solution of the trajectory equation

$$
\frac{d x}{d t}=u, \quad x(X, 0)=X .
$$

Here, $x$ stands for the Eulerian coordinate. Expressing the fluid variables in terms of the Eulerian coordinate and noticing that mass conservation allows us to write $\mathrm{d} m=\rho^{0}(X) \mathrm{d} X=\rho(x(X, t), t) \mathrm{d} x$, where $\rho^{0}=\rho^{0}(X)>$ 0 is the initial density. The $1 \mathrm{D}$ system of hydrodynamics Euler equations can be written as

$$
\begin{aligned}
& \rho \frac{d}{d t}\left(\frac{1}{\rho}\right)-\frac{\partial u}{\partial x}=0, \\
& \rho \frac{d}{d t} u+\frac{\partial P}{\partial x}=0, \\
& \rho \frac{d}{d t} E+\frac{\partial}{\partial x}(P u)=0,
\end{aligned}
$$

where $\frac{d}{d t}=\frac{\partial}{\partial t}+u \frac{\partial}{\partial x}$ denotes the material derivative. The specific internal energy $\varepsilon$ is defined by $\varepsilon=$ $E-\frac{1}{2} u^{2}$. The thermodynamic closure is given by the equation of state (EOS) $P=P(\rho, \varepsilon)$. To ensure the thermodynamic consistency of the above system with the Second Law of thermodynamics, it must be completed by the following entropy inequality $\rho \frac{d \eta}{d t} \geq 0$, where $\eta$ denotes the specific entropy. The specific entropy is related to the other thermodynamic variables by means of the fundamental Gibbs relation $T \mathrm{~d} \eta=\mathrm{d} \varepsilon+P \mathrm{~d}\left(\frac{1}{\rho}\right)$, where $T$ denotes the fluid temperature.

\footnotetext{
${ }^{1}$ Note that most of this section has been freely inspired by the chapter 4 of [58].
} 


\subsection{Lagrangian scheme}

\subsubsection{First order discretization}

Let $\Omega=\left[X_{\min }, X_{\max }\right]$ be the domain initially filled with the fluid. This domain is partitioned into $N_{c}$ cells $\Omega_{i}=\left[X_{i-\frac{1}{2}}, X_{i+\frac{1}{2}}\right]$, for $1 \leq i \leq N_{c}$. The vertex motion is described by the discretized trajectory equation

$$
\frac{d}{d t} x_{i+\frac{1}{2}}=u_{i+\frac{1}{2}}, \quad x_{i+\frac{1}{2}}(0)=X_{i+\frac{1}{2}}
$$

where $u_{i+\frac{1}{2}}$ denotes the vertex velocity. Let $I_{i}(t)=\left[x_{i-\frac{1}{2}}(t), x_{i+\frac{1}{2}}(t)\right]$ denote the Eulerian moving cell, corresponding to the Lagrangian cell $\Omega_{i}$ in the flow map. Let $\Phi_{i}=\left(\frac{1}{\rho_{i}}, u_{i}, E_{i}\right)$ be the mass averaged values of $\left(\frac{1}{\rho}, u, E\right)$ over the cell $I_{i}(t)$. We recall that in the Lagrangian framework, the mass $m_{i}$ of the cell $I_{i}(t)$ is constant

$$
m_{i}=\rho_{i}(t) \Delta x_{i}(t), \quad \forall t>0,
$$

where $\Delta x_{i}=x_{i+\frac{1}{2}}-x_{i-\frac{1}{2}}$ denotes the volume of the cell. Using the transport formula, the integration of (13) over $I_{i}$ leads to the following set of evolution equations for the discrete variables $\Phi_{i}=\left(\frac{1}{\rho_{i}}, u_{i}, E_{i}\right)$

$$
\begin{aligned}
& m_{i} \frac{d}{d t}\left(\frac{1}{\rho_{i}}\right)-\left(u_{i+\frac{1}{2}}-u_{i-\frac{1}{2}}\right)=0, \\
& m_{i} \frac{d}{d t} u_{i}+P_{i+\frac{1}{2}}-P_{i-\frac{1}{2}}=0, \\
& m_{i} \frac{d}{d t} E_{i}+(P u)_{i+\frac{1}{2}}-(P u)_{i-\frac{1}{2}}=0,
\end{aligned}
$$

where $u_{i+\frac{1}{2}}, P_{i+\frac{1}{2}}$ and $(P u)_{i+\frac{1}{2}}$ are the numerical fluxes at node $x_{i+\frac{1}{2}}$ under the fundamental assumption that $(P u)_{i+\frac{1}{2}}=P_{i+\frac{1}{2}} u_{i+\frac{1}{2}}$. The fluxes $u_{i+\frac{1}{2}}$ and $P_{i+\frac{1}{2}}$ are obtained exactly or approximately by solving the Riemann problem at the cell interface $x_{i+\frac{1}{2}}$ for the left state $\Phi_{l}=\left(\frac{1}{\rho_{i}}, u_{i}, E_{i}\right)^{t}$ and the right state $\Phi_{r}=\left(\frac{1}{\rho_{i+1}}, u_{i+1}, E_{i+1}\right)^{t}$. In our case, we choose the acoustic approximate Riemann solver, otherwise called Godunov acoustic solver [9]. This solver is cheap and versatile since its use only requires the knowledge of the isentropic sound speed and can handle tabulated equations of state. The acoustic solution of the Riemann problem defined by states $\Phi_{l}, \Phi_{r}$ is given by

$$
u^{*}=\frac{z_{l} u_{l}+z_{r} u_{r}}{z_{l}+z_{r}}-\frac{P_{r}-P_{l}}{z_{l}+z_{r}}, \quad P^{*}=\frac{z_{l} P_{r}+z_{r} P_{l}}{z_{l}+z_{r}}-\frac{z_{l} z_{r}}{z_{l}+z_{r}}\left(u_{r}-u_{l}\right),
$$

where $z_{s}$ is the acoustic impedance, $z_{s}=\rho_{s} a_{s}$, with $a_{s}$ is the local sound speed.

The first order in time discretization assumes that all fluid variables are cell-centered and known at time $t=t^{n}$. Classically we denote them with the superscript $n$ and the time-step $\Delta t=t^{n+1}-t^{n}$. A standard forward Euler scheme discretization of system (16) yields

$$
\begin{aligned}
& m_{i}\left(\frac{1}{\rho_{i}^{n+1}}-\frac{1}{\rho_{i}^{n}}\right)-\Delta t\left(u_{i+\frac{1}{2}}^{n}-u_{i-\frac{1}{2}}^{n}\right)=0, \\
& m_{i}\left(u_{i}^{n+1}-u_{i}^{n}\right)+\Delta t\left(P_{i+\frac{1}{2}}^{n}-P_{i-\frac{1}{2}}^{n}\right)=0, \\
& m_{i}\left(E_{i}^{n+1}-E_{i}^{n}\right)+\Delta t\left(P_{i+\frac{1}{2}}^{n} u_{i+\frac{1}{2}}^{n}-P_{i-\frac{1}{2}}^{n} u_{i-\frac{1}{2}}^{n}\right)=0,
\end{aligned}
$$

where $u_{i+\frac{1}{2}}^{n}$ and $P_{i+\frac{1}{2}}^{n}$ are the fluxes at node $x_{i+\frac{1}{2}}$ obtained by solving the Riemann problem for the left state $\Phi_{l}=\left(\frac{1}{\rho_{i}^{n}}, u_{i}^{n}, E_{i}^{n}\right)^{t}$ and the right state $\Phi_{r}=\left(\frac{1}{\rho_{i+1}^{n}}, u_{i+1}^{n}, E_{i+1}^{n}\right)^{t}$.

The previous set of discrete equations (18) leads to a first order accurate scheme both in space and time. In addition, the vertex motion is simply given by

$$
x_{i+\frac{1}{2}}^{n+1}=x_{i+\frac{1}{2}}^{n}+\Delta t u_{i+\frac{1}{2}}^{n}
$$


with $x_{i+\frac{1}{2}}^{0}=X_{i+\frac{1}{2}}$, corresponding to the discretization of (14). To complete the Lagrangian phase, we must evaluate the new specific internal energy, which is done as $\varepsilon_{i}^{n+1}=E_{i}^{n+1}-\frac{1}{2}\left(u_{i}^{n+1}\right)^{2}$. Finally, using the EOS, the pressure $P_{i}^{n+1}=P\left(\rho_{i}^{n+1}, \varepsilon_{i}^{n+1}\right)$, the sound speed $a_{i}^{n+1}$ and the acoustic impedance $z_{i}^{n+1}$ are computed at the new time level.

The time step is evaluated following the Courant Friedrich Levy (CFL) stability condition [1]. At time $t^{n}$, we compute

$$
\Delta t=\mathrm{CFL} \min _{i} \frac{\Delta x_{i}^{n}}{a_{i}^{n}}
$$

where CFL is a strictly positive coefficient and $a_{i}^{n}$ is the sound speed in the cell $\Omega_{i}$. Knowing $\Delta t^{n-1}$, the evaluation of the next time step $\Delta t^{n}$ is performed as follows

$$
\Delta t^{n}=\min \left(\Delta t, C_{M} \Delta t^{n-1}, \Delta t_{v}\right),
$$

where $C_{M}$ is a multiplicative coefficient, which allows the time step to increase moderately. Moreover, we add a criterion on the cell volume variation so that $\Delta t_{v}$ is such that $\frac{\left|v_{i}^{n+1}-v_{i}^{n}\right|}{v_{i}^{n}} \leq C_{v}$ where $C_{v}$ is a strict positive constant less than one and $v_{i}^{n}$ refers to the cell volume at time $t^{n}$. For numerical applications, we generally use CFL $=0.25, C_{M}=1.01$ and $C_{v}=0.1$, such as recommended in [56].

\subsubsection{Second order discretization}

Here, we summarize the derivation of a high-order extension of the previous scheme [60] using a predictor-corrector time discretization and Monotonic Upstream-centered Schemes for Conservation Laws (MUSCL) like spacial reconstructions following the pioneering works of van Leer [33] and Kolgan [61, 62]. Let us assume a piece-wise linear representation of pressure and velocity at time $t^{n}$, that is, for all $x \in$ $\left[x_{i-\frac{1}{2}}^{n}, x_{i+\frac{1}{2}}^{n}\right]$,

$$
\widetilde{u}_{i}(x)=u_{i}^{n}+\phi_{i}^{u}(\delta u)_{i}^{n}\left(x-x_{i}^{n}\right), \quad \widetilde{P}_{i}(x)=P_{i}^{n}+\phi_{i}^{P}(\delta P)_{i}^{n}\left(x-x_{i}^{n}\right),
$$

where $x_{i}^{n}=\frac{1}{2}\left(x_{i-\frac{1}{2}}^{n}+x_{i+\frac{1}{2}}^{n}\right)$ is the midpoint of cell $I_{i}^{n}=\left[x_{i-\frac{1}{2}}^{n}, x_{i+\frac{1}{2}}^{n}\right]$, and $(\delta u)_{i}^{n}$ and $(\delta P)_{i}^{n}$ are the slopes of velocity and pressure that can be determined using for instance a least squares method considering $i-1$ and $i+1$ as neighbors. A slope limiter $\phi_{i}$ is supplemented to reduce the slope value to avoid formation of new extremal values. There is a vast literature about slope/flux limiters, some of them are now known after their discoverers (van Leer [31, 32, 33], van Albada [34], Sweby [35], Barth-Jespersen [36], Venkatakrishnan [37], Koren [38, 39], etc.) or their particularity (minmod [40], superbee [40], monotonized central [32], etc.). Such classical limiters all rely on a priori information, that is to say the information at $t^{n}$ before the actual step. It is important to note that their role is twofold: (i) it must detect if some cell reconstructions will lead to unacceptable values at $t^{n+1}$, and, in that case, (ii) it must correct the situation in order to ensure that the final numerical solution is valid.

For instance, when the so called Barth-Jespersen (BJ) limiter is used then the slope of the $\mathbb{P}_{1}$ polynomial $\widetilde{u}_{i}$ is reduced so that the reconstructed states on the left and right boundaries of cell $\Omega_{i}$ are in bounds. The bounds are defined by the minimal and maximal values of the constant data $u_{j}^{n}$ for $j$ in the direct neighborhood surrounding $\Omega_{i}$, that is $B_{i}^{n}=\max _{j=i \pm 1, i}\left(u_{j}^{n}\right)$ and $b_{i}^{n}=\min _{j=i \pm 1, i}\left(u_{j}^{n}\right)$.

The limiter $\phi_{i}^{u}$ is computed such that the following constraints are fulfilled

$$
b_{i}^{n} \leq \widetilde{u}_{i}\left(x_{i-1 / 2}^{n}\right) \leq B_{i}^{n}, \quad \text { and } \quad b_{i}^{n} \leq \widetilde{u}_{i}\left(x_{i+1 / 2}^{n}\right) \leq B_{i}^{n},
$$

likewise for $\phi^{P}$. In this work, the Barth-Jespersen [36] or Venkatakrishnan [37] limiter is used.

Then, using these second order limited reconstructions, we can evaluate more accurately the left and right states at point $x_{i+\frac{1}{2}}^{n}$ to feed our acoustic Riemann solver with the state values $\widetilde{u}_{i}\left(x_{i \pm \frac{1}{2}}^{n}\right), \widetilde{P}_{i}\left(x_{i \pm \frac{1}{2}}^{n}\right)$. Recall 
that the acoustic Riemann solver only demands the knowledge of the impedance, $z_{i}$ and $z_{i+1}$. These can be in the simplest case considered constant during the time-step and equal to $z_{i}^{n}=\rho_{i}^{n} a_{i}^{n}$ and $z_{i+1}^{n}=\rho_{i+1}^{n} a_{i+1}^{n}$, however in practice, modified version described in [63] is used and further supplemented with the left and right velocity/pressure reconstructed states, recall (17).

The generic high-order Godunov-type cell-centered Lagrangian scheme of second-order in time can be written under the general form

$$
\begin{aligned}
m_{i}\left(\frac{1}{\rho_{i}^{n+1}}-\frac{1}{\rho_{i}^{n}}\right)-\Delta t\left(u_{i+\frac{1}{2}}^{n+\frac{1}{2}}-u_{i-\frac{1}{2}}^{n+\frac{1}{2}}\right) & =0, \\
m_{i}\left(u_{i}^{n+1}-u_{i}^{n}\right)+\Delta t\left(P_{i+\frac{1}{2}}^{n+\frac{1}{2}}-P_{i-\frac{1}{2}}^{n+\frac{1}{2}}\right) & =0, \\
m_{i}\left(E_{i}^{n+1}-E_{i}^{n}\right)+\Delta t\left((P u)_{i+\frac{1}{2}}^{n+\frac{1}{2}}-(P u)_{i-\frac{1}{2}}^{n+\frac{1}{2}}\right) & =0 .
\end{aligned}
$$

supplemented with the discrete grid motion which is governed by the discretized trajectory equation

$$
x_{i+\frac{1}{2}}^{n+1}=x_{i+\frac{1}{2}}^{n}+\Delta t u_{i+\frac{1}{2}}^{n+\frac{1}{2}}
$$

Here, $u_{i+\frac{1}{2}}^{n+\frac{1}{2}}, P_{i+\frac{1}{2}}^{n+\frac{1}{2}}$ and $(P u)_{i+\frac{1}{2}}^{n+\frac{1}{2}}$ are the time-averaged numerical fluxes at node $x_{i+\frac{1}{2}}$ over the time interval $\left[t^{n}, t^{n+1}\right]$. Several classical techniques are available to estimate these time-averaged numerical fluxes based on iterations (predictor/corrector scheme, Runke-Kutta scheme, etc.), or more advanced approaches based on one step integration methods (Generalized Riemann Problem GRP [64, 65, 66, 58, 56], Arbitrary high order DERivatives ADER [67, 68, 69, 70], etc.). In this work we use the GRP version, see [58, 56] for details and some analysis of this scheme.

Let us remark, that this nominally second-order cell-centered Lagrangian scheme does not require any artificial viscosity like term, because the approximate Riemann solver along with the slope limiter provide some embedded and (usually) sufficient numerical dissipation.

\section{High-accurate indirect ALE scheme}

In this section, we simply and briefly describe the inter-connection of a Lagrangian scheme with rezone and remap procedures to produce an indirect ALE scheme. Depending on the chosen rezone strategy, which determines the target mesh for the remapper, the overall scheme can be either

Lagrangian: if the target mesh is the Lagrangian mesh (no remapping is needed);

Eulerian: as Lagrange+Remap $(\mathrm{L}+\mathrm{R})$, if the rezone procedure constantly selects the initial mesh onto which the remap operates;

ALE: for any other choice of target mesh, assuming that the Lagrangian and rezone meshes pave the same computational domain.

For the sake of generality, we assume that the rezoning strategy provides a target mesh $\mathcal{T}$ that has a priori no logical connection with $O$, the Lagrangian/original one. In this section, we first present the classical secondorder accurate remap scheme. Then in the second time we introduce our more accurate version based on high-order polynomial and hyperbolic tangent (THINC) function reconstructions [44, 45, 46, 47, 48, 49] supplemented with an interface tracking algorithm. 


\subsection{Classical second-order remap scheme: piece-wise linear reconstruction + slope limiter}

The indirect ALE scheme starts from cell-centered data at time $t^{n}, \Phi_{i}^{n}=\left(\frac{1}{\rho_{i}^{n}}, u_{i}^{n}, E_{i}^{n}\right)$, in each cell $I_{i}^{n}=$ $\left[x_{i-\frac{1}{2}}^{n}, x_{i+\frac{1}{2}}^{n}\right]$ from the original mesh $O^{n}$.

Lagrangian scheme The nominally second-order Lagrangian scheme determines the time-step $\Delta t$ and updates the state and geometrical variables $\Phi_{i}^{n+1}, x_{i+\frac{1}{2}}^{n+1}$ up to time $t^{n+1}$. During this time-step, the mesh has moved with the fluid velocity and $I_{i}^{n+1}=\left[x_{i-\frac{1}{2}}^{n+1}, x_{i+\frac{1}{2}}^{n+1}\right]$ for every cell $i$, and the whole mesh is called $\mathrm{O}^{n+1}$.

Rezone strategy In the second step, the rezone stage, the target mesh $\mathcal{T}$ is set. In other words, the rezone procedure defines $\widetilde{x}_{j+\frac{1}{2}}^{n+1}$ for any cell $j=1, \ldots, J$ as being the new point position determining the target cells $J_{j}^{n+1}$. Therefore, for the remap stage, we indeed consider the intersection of cell $I_{i} \equiv I_{i}^{n+1}$ and $J_{j} \equiv J_{j}^{n+1}$ which we have called $K_{i, j} \equiv K_{k}$ in section 2 .

Remapping procedure Then the remap algorithm operates to produce the cell-centered conserved state variables $\widetilde{\Phi}_{j}^{n+1}$ in cells $J_{j}^{n+1}$ for all $j=1, \ldots, J$. It uses the cell-centered piece-wise linear limited reconstructions from the original mesh to enhance the accuracy of the remapping without sacrificing thou bound preservation property. As a consequence, the mean values are located at the cell-centers of the target mesh $\mathcal{T}^{n+1}$ at time $t^{n+1}$.

In the classical second-order remapping, a least-squares procedure fits at best the slope of the piece-wise linear reconstruction with the mean values in the two neighboring cells of the current one. However, let us present the polynomial reconstruction in a more general sense. Starting from the mean values $a_{i}$ of a smooth enough function $a(x)$ over cell $I_{i}$ of an arbitrary mesh, we define the neighborhood of $I_{i}$ as the set of $p$ and $q$ cells to its left and right sides: $\mathcal{S}_{i}^{p, q}=\left\{I_{k}, k=i-p, \cdots, i+q\right\}$. With the associated $p+q$ neighbor mean values, we can define the polynomial of degree $m$ in cell $i$ as $\widehat{a}_{i}(x)=\sum_{j=0}^{m} \widehat{a}_{j} x^{j}$, where $m \leq(p+q)$. Usually, we choose $p=q$. The unknown coefficients $\widehat{a}_{j}$ are further determined to fit $\widehat{a}_{i}(x)$ to the data $a_{k}$ from the neighborhood $\mathcal{S}^{p, q}$ in the least-squares sense under the constraint of conservation of local mean value, that is

$$
\arg \min _{\widehat{a}_{k}}\left|\frac{1}{\left|I_{k}\right|} \int_{I_{k}} \widehat{a}_{i}(x) d x-a_{k}\right|^{2} \text {, under constraint } \frac{1}{\left|I_{i}\right|} \int_{I_{i}} \widehat{a}_{i}(x) d x=a_{i} .
$$

This problem has always at least one solution because the number of degrees of freedom is less or equal than the number of data. Following [71], this over-determined system can be solved by the QR decomposition with the Householder transformation [72]. We have then defined the operator R of (9) which works for the set of polynomials of degree $m>0$. For instance, for the second-order (linear) reconstruction, we set $m=1$. Once the slope $\widehat{a}_{1}$ is computed, then a limiter is activated like the one described in the Lagrangian step, see section 3.2.2. Usually, the Barth and Jespersen slope limiter [36] is coupled with this reconstruction.

\subsection{High accurate remapping scheme}

In this section, we describe the process combining several reconstruction techniques into one remapping scheme. In general, any suitable function can be used for the reconstruction in the particular cell. In this work, we combine quartic polynomials $\mathbb{P}_{4}$, limited linear function $\mathbb{P}_{1}^{\text {lim }}$, and the non-polynomial hyperbolic tangent THINC reconstructions. 


\subsubsection{High accuracy $\mathbb{P}_{4}:$ Quartic polynomial reconstruction}

Let us define the neighborhood of cell $I_{i}$ as the set of two neighbor cells to the left and to the right: $\mathcal{S}_{i}^{2,2}=\left\{I_{k}, k=i-2, \cdots, i+2\right\}$, that is with $m=2$. With the associated stencil of 5 cells and their mean values $a_{k}$, we can define the quartic polynomial in cell $I_{i}$ as $\widehat{a}_{i}(x)$. The unknown coefficients $\widehat{a}_{j}$ are further determined with the previously described operator R (least-square procedure (27)). Note that a 5 th order of accuracy is obtained if the underlying function is smooth enough. On the contrary, this reconstruction does oscillate in the case of discontinuous or irregular one. Unfortunately, there exists no agreement on how to limit high order polynomial reconstructions. As a consequence, such accurate but un-safe reconstruction will be our preferred choice in most of the domain, where the solution is smooth enough.

\subsubsection{Non-oscillatory $\mathbb{P}_{1}$ : Piece-wise linear polynomial reconstruction with slope limiter}

By setting $m=1$ we can also re-use the linear reconstruction with limiter summarized in section 4.1. This is a safe choice when the underlying solution is irregular. This will our preferred reconstruction in this later case.

\subsubsection{Interface sharpening THINC : Non-linear hyperbolic tangent reconstruction}

For a strictly increasing or decreasing set of mean values $a_{i-1}, a_{i}, a_{i+1}$, the THINC (Tangent Hyperbola for INterface Capturing method) $[44,45,46,47,48,49]$ function in cell $I_{i}$ is given by

$$
q_{i}(x)=a_{\min }+\frac{[[a]]}{2}\left[1+\theta \tanh \beta\left(\frac{x-x_{i-1 / 2}}{x_{i+1 / 2}-x_{i-1 / 2}}-\tilde{x}_{i}\right)\right],
$$

where we define the values

$$
a_{\min }=\min _{x \in \mathcal{N}_{i}}(a(x)), \quad a_{\max }=\max _{x \in \mathcal{N}_{i}}(a(x)), \quad[[a]]=a_{\max }-a_{\min }, \quad \theta=\operatorname{sgn}\left(a_{i+1}-a_{i-1}\right),
$$

where $\mathcal{N}_{i}$ is the neighborhood in the vicinity of cell $I_{i}$. Classically, the min and max value are defined for Eulerian FV schemes using THINC [48, 49] by $a_{\min }=\min \left(a_{i-1}, a_{i+1}\right)$ and $a_{\max }=\max \left(a_{i-1}, a_{i+1}\right)$ on a stencil $\mathcal{N}_{i}$ made of two cells. However, in our remapping context, those maximal and minimal values are evaluated thanks to piece-wise linear limited polynomial reconstructions in cells $i-1$ and $i+1$, that is

$$
a_{\min }=\min \left(\widehat{a}_{i-1}\left(x_{i-1 / 2}\right), \widehat{a}_{i+1}\left(x_{i+1 / 2}\right)\right), \quad a_{\max }=\max \left(\widehat{a}_{i-1}\left(x_{i-1 / 2}\right), \widehat{a}_{i+1}\left(x_{i+1 / 2}\right)\right) .
$$

This choice provides more smooth reconstruction profile across the cell boundaries, which in practice prevents degeneration of the contact resolution. The parameter $\beta$ controls the jump thickness and $\widetilde{x}_{i}$ represents the relative location of the jump center, see figure 1 . It is obtained by enforcing the conservation of the mean value, ie $a_{i}=\frac{1}{\Delta x_{i}} \int_{I_{i}} q_{i}(x) d x$. Substituting (28) into this conservation relation leads to a non-linear equation, which can still be solved analytically. The value of $\widetilde{x}_{i}$ can be expressed [46] in a unique and explicit form:

$$
\tilde{x}_{i}=\frac{1}{2 \beta} \ln \left(\frac{\exp \left(\frac{\beta}{\theta}\left(1+\theta-2 a_{i}\right)\right)}{1-\exp \left(\frac{\beta}{\theta}\left(1-\theta-2 a_{i}\right)\right)}\right) .
$$

In other words, this choice of $\widetilde{x}_{i}$ makes THINC reconstruction (28) a conservative one. Let us remark that the stencil is reduced to few neighbors which are solely used to determine the bounds $a_{\min }, a_{\max }$ into which the THINC function is restricted. Hence a THINC function is 'in-bounds' by construction. Notice that if the mean values $a$ are almost flat, so will be such reconstruction. Moreover, if the mean values are not monotonically increasing or decreasing, then we can not reconstruct a THINC function. Instead we set 


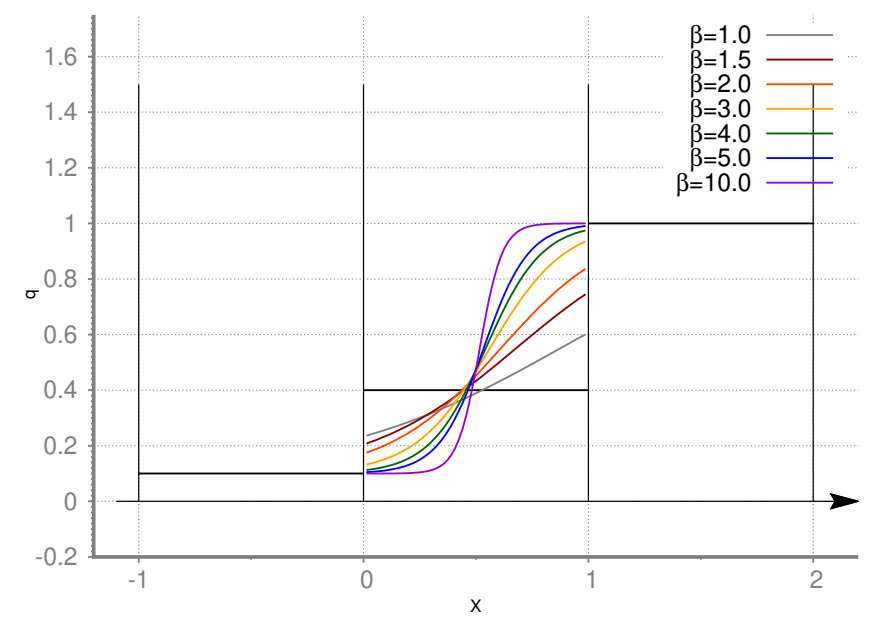

Figure 1: Illustrative examples of THINC functions $q$ parameterized by the jump thickness $\beta$ computed from cell mean values $\left(a_{i-1}, a_{i}, a_{i+1}\right)=(0.1,0.4,1.0)$.

$q_{i}(x)=a_{i}$ for all $x$. In our remap case we employ $\beta=15$ which makes the THINC function relatively steep, see figure 1 where we present some illustrative examples of THINC reconstructions starting from cell mean values $\left(a_{i-1}, a_{i}, a_{i+1}\right)=(0.1,0.4,1.0)$ for $\Delta x=1$.

As a summary, the THINC function is a non-linear reconstruction that connects minimum and maximum values by a hyperbolic tangent. It is designed to be used for cells in which a true discontinuity is located, such as a contact. In such cells, any high order polynomial reconstruction do oscillate, while the THINC reconstruction is prone to produce a genuine accurate representation.

\subsection{Interface tracking algorithm}

We need to determine an algorithm, called 'interface detector' or 'interface tracking' which ranks the cells according to the reconstruction types: THINC, $\mathbb{P}_{4}$ or $\mathbb{P}_{1}$ lim . Ideally $\mathbb{P}_{4}$ is employed when the solution is genuinely smooth, $\mathbb{P}_{1}^{\text {lim }}$ when there is a doubt on regularity, for instance in the vicinity of shocks, and, THINC if the cell contains an interface.

Tracking material interface and contact. A Lagrangian scheme preserves the contacts by construction when they are located on cell boundaries. These contacts separate two different materials or different states of the same material. In the hydrodynamics context it usually translates into a jump in density, while velocity and pressure remain constant. Because of the constant fluid velocity, the left and right cells move with the same velocity, and, therefore they remain "pure".

One drawback of the remap stage of an indirect ALE scheme is the mixing of those two states if the pure cells are not maintained by the rezone mesh on which the remap operates. In our approach, if we track the cells in which such discontinuities are, then a THINC reconstruction could be used inhere. Although the same diffusion process will occur because we create a mixed cell, one expects that its magnitude will be drastically lowered due this more accurate discontinuity representation via THINC. Hence, our tracking algorithm initially marks the discontinuity $y_{i}^{0}$ in cell $I_{i}^{0}$ containing a discontinuity (or the cells surrounding one). This tracked contact position is updated with the fluid velocity during the Lagrangian steps as

$$
y_{i}^{n+1}=y_{i}^{n}+\Delta t\left(\left(1-\alpha_{i}\right) u_{i-\frac{1}{2}}^{n+\frac{1}{2}}+\alpha_{i} u_{i+\frac{1}{2}}^{n+\frac{1}{2}}\right), \quad \alpha_{i}=\frac{y_{i}^{n}-x_{i-\frac{1}{2}}^{n}}{\Delta x_{i}^{n}} .
$$

Once a remap stage is called for instance at time $t^{n+1}$, then the THINC reconstruction is computed in the old cell $I_{i}^{n+1}$ welcoming a contact. This position is obviously fixed during the remap stage because it is 
just a conservative interpolation between meshes, therefore we can determine in which rezoned cell $J_{j}$ it is located. This cell $J_{j}$ (in the new mesh) is the one in which the discontinuity remains for the next successive Lagrangian steps.

Tracking compression area. A shock wave is typically spread by the Lagrangian scheme over two to four cells, depending on the embedded numerical viscosity. A second-order remap scheme usually does not overly degrade nor improve this situation and we do not expect to improve this situation. Therefore, we want to use piece-wise linear limited reconstructions in the vicinity of compression area. To detect such area, we rely on a type of artificial viscosity switch from the standard staggered Lagrangian scheme. More precisely we state that a cell endures a 'high' compression if its volume evolved during a time-step as: $v_{i}^{n+1}<\kappa v_{i}^{n}$ where $0<\kappa<1$ is a user given parameter. Manipulating this equation yields

$$
\left(u_{i+\frac{1}{2}}^{n+\frac{1}{2}}-u_{i+\frac{1}{2}}^{n+\frac{1}{2}}\right)<(\kappa-1) \frac{v_{i}^{n}}{\Delta t}
$$

(31) is the detection criteria used to determine if cell $I_{i}$ is under compression. If so, it demands that a safe reconstruction is used in its vicinity. The vicinity of cell $I_{i}$ is defined as the neighborhood made by two cells to the left and right, for which the $\mathbb{P}_{1}$ reconstruction supplemented with a slope limiter is employed. We use $\kappa=0.95$ in our simulations, corresponding to $5 \%$ cell compression to be detected as shock cell. We are aware that this compression detector is not unique and far from perfect. However, it is simple and can be easily replaced by a different shock detector, adjusted for the particular application.

Regular solution. At last, any cell which has not been marked or tracked by the previous algorithm is considered as a regular one. In these cells, we can achieve the maximal accuracy by using the unlimited $\mathbb{P}_{4}$ polynomial reconstruction.

Note that all conservative variables are treated alike, that is with the same reconstruction type, should it be $\mathbb{P}_{4}, \mathbb{P}_{1}^{\text {lim }}$ or THINC, because combining different reconstructions in a single cell typically results in spurious oscillations due to inconsistent representation of fluid quantities.

\subsection{Summary of the indirect ALE scheme}

The main steps of the 1D cell-centered indirect ALE scheme with the contact-tracking and shockdetecting algorithm introduced in section 4.3 are briefly summarized as:

1. Lagrangian scheme:

(a) Compute cell acoustic impedances by the approach suggested in [63].

(b) Perform piece-wise linear reconstruction of velocity and pressure in each cell (21), employing least-squares and Venkatakrishnan limiter [37].

(c) Using the Riemann solver (17), compute the central state velocity and pressure.

(d) Calculate time step size (20).

(e) By the GRP scheme [64, 65, 66], approximate central state temporal derivatives, and use them to estimate nodal pressure and velocity at time level $t^{n+\frac{1}{2}}$.

(f) Update cell density, velocity, and total energy by (23)-(25), and update the nodal positions by (26).

(g) Update cell volumes and centroids.

(h) Propagate contact positions in the cell, using linear interpolation of nodal velocities at time level $t^{n+\frac{1}{2}}(30)$.

(i) Compute velocity divergence from nodal velocities at $t^{n+\frac{1}{2}}$ and remember it for the shock detector (31).

2. Rezone scheme: determine the target mesh $\mathcal{T}$.

3. Remap scheme: all cell-centered conservative values $\left(\rho_{i},(\rho u)_{i},(\rho E)_{i}\right)$ defined on the original/Lagrangian mesh are transferred onto the target/rezone mesh

(a) Mark cells by reconstruction type as discussed in section 4.3: 
- if a tracked contact belongs to a cell, mark is as THINC, and mark two layers of neighbors as $\mathbb{P}_{1}^{\lim \text {; }}$

- if the shock detector (31) is on, mark the cell as $\mathbb{P}_{1}^{\text {lim }}$, and again mark also two layers of neighbors as $\mathbb{P}_{1}^{\text {lim }}$;

- mark the rest of the cell as $\mathbb{P}_{4}$.

(b) From the primitive variables, compute cell-centered density of mass, momentum, and total energy.

(c) Perform reconstruction of each quantity according to the marker, so different reconstructions are used in different cells, as discussed in sections 4.2.1-4.2.3. The same reconstruction type is used for all quantities in a particular cell.

(d) Construct the intersections of Lagrangian and rezoned mesh, and integrate the reconstructions by a high-order quadrature rule (11).

(e) Compute new cell masses, velocities, and internal energies in a standard way. Use EOS do compute new pressures needed for the next Lagrangian step.

The described algorithm allows to combine the cell-centered Lagrangian solver with rezoning/remapping steps, including tracking and detection of problematic cells, allowing to perform different types of reconstructions in different cells during a single ALE step. In the next section, we will present the numerical results obtained with this indirect ALE scheme.

\section{Numerical experiments for static reconstruction and remap}

In this section, we illustrate and test the high accurate reconstruction procedure and remap scheme for static situations, when we analytically follow or track the static discontinuities through the moving mesh. The purpose is to test the remapping strategy using mixed reconstructions without using the interface detector from section 4.3. Then in the next section, for the hydrodynamics test cases, the interface detector will be used.

\subsection{Static reconstruction}

In this section we ought to illustrate the reconstruction procedure. Let us consider a domain $[-1,1]$ mesh with 5 equidistant cells and a step-like function

$$
\rho(x)= \begin{cases}2 & \text { if } x \leq x_{d} \\ 1 & \text { else }\end{cases}
$$

The discontinuity is set at position $x_{d}=0$ in the central cell and the exact mean values are $\rho_{i=1,2,3,4,5}=$ $(2,2,1.5,1,1)$ using $\mathbb{P}_{4}, \mathbb{P}_{1}, \mathbb{P}_{1}^{\text {lim }}$ and THINC function. The mesh is extended with ghost cells for the high order polynomial reconstructions. The results are plotted in figure 2. As expected, the polynomial reconstructions without limiting produce out-of-bounds values, while the use of a slope limiter prevents the $\mathbb{P}_{1}$ reconstruction from such drawbacks. The last panel presents THINC reconstruction in the central cell and left/right shifted reconstruction stencils for $\mathbb{P}_{4}$ for the second/fourth cells respectively. As observed, such mixed reconstruction can nicely reproduce a step-like function. In figure 3, we present the same reconstructions but with the discontinuity located closer to the cell boundary, at $x_{d}=0.19$. The same conclusion applies.

\subsection{Static remap}

The classical test case for remapping scheme consists of successive remap steps of a given static profile under a non uniform mesh motion. It can be found under the name "cyclic remapping" in [26] for instance. This process starts from an initial 1D uniform grid $\left(\Omega=[-1,1], N_{p}\right.$ nodes, $N_{c}=N_{p}-1$ cells $)$ on which a 


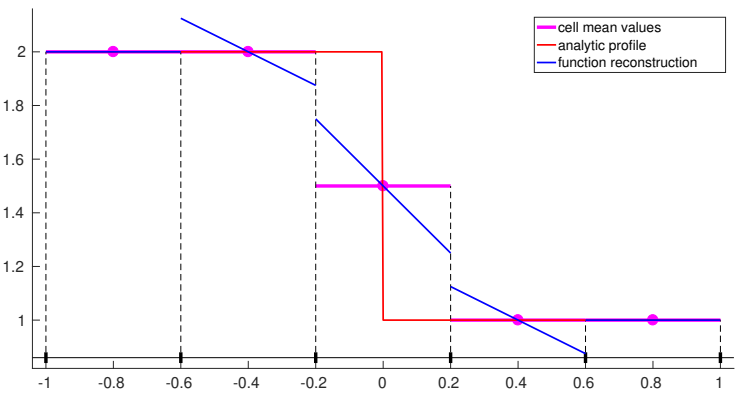

(a) $\mathbb{P}_{1}$

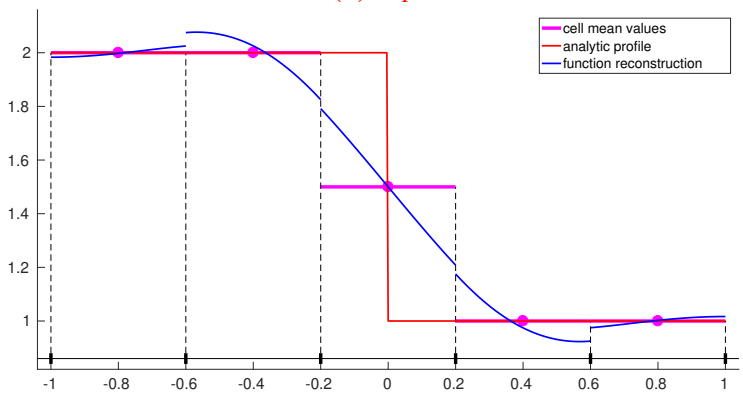

(c) $\mathbb{P}_{4}$

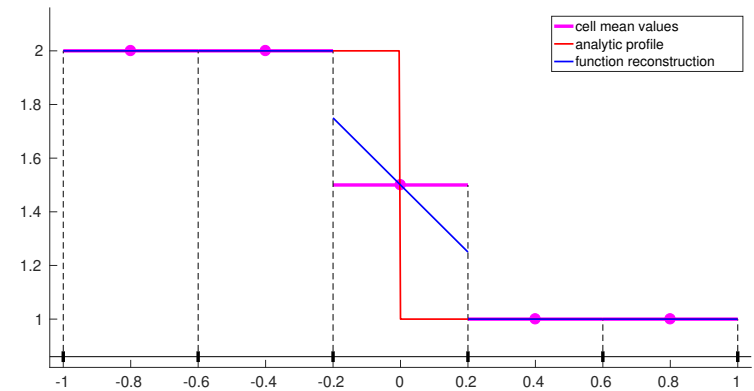

(b) $\mathbb{P}_{1}^{\text {lim }}$

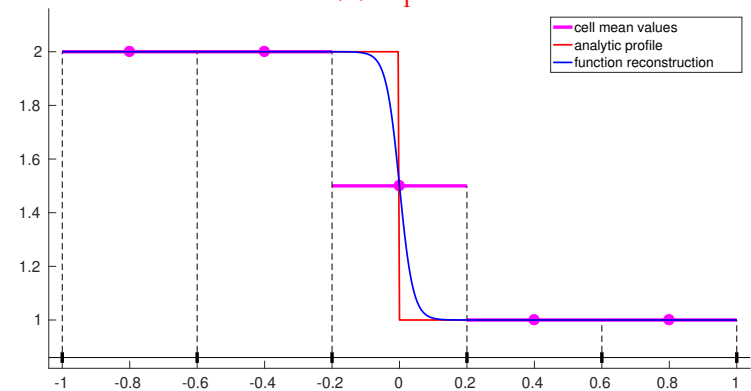

(d) THINC

Figure 2: Static reconstruction problem of a step like function at position $x_{d}=0$. Different reconstructions are shown: $\mathbb{P}_{1}$ (a), $\mathbb{P}_{1}^{l i m}$ (b), $\mathbb{P}_{4}$ (c) and THINC (d). The last panel presents THINC reconstruction in the central cell and left/right shifted stencils for $\mathbb{P}_{4}$ reconstructions for the second/fourth cells respectively.

"shape" $\rho$ is given by its cell-centered mean values. Then a non-uniform mesh motion is applied for each time step described by the following formula

$$
x_{i+1 / 2}^{n}=\left(1-\alpha_{n}\right) \xi_{i+1 / 2}+\alpha_{n} \xi_{i+1 / 2}^{3},
$$

where $\xi_{i}=\frac{i-1}{N_{p}-1}$ and $\alpha_{n}=\frac{1}{2} \sin \left(\frac{4 \pi n}{N_{\max }}\right)$, and $0 \leq n \leq N_{\max }=5 N_{c}$ is the time-step index. Note that for iterations $n=0$ and $n=N_{\max }$, we have $\alpha^{n}=0$ and therefore $x_{i}^{0}=x_{i}^{N_{\max }}$, so we end up with the same initial uniform grid. In each time step, a function $\rho$ defined on the old grid defined by points $x^{n}$ is remapped onto the new one defined by $x^{n+1}$. Finally, a comparison between the initial mean values and the final ones allows to assess the accuracy, robustness and behavior of the remap scheme.

A set of four different smooth and irregular shapes are considered, a Gaussian, a rectangular and triangular shapes and a parabola, see [73]. In a concise way, the four shape profile [74] is defined as

$$
\rho(x)=1+ \begin{cases}1+(G(x, \beta, z-\delta)+G(x, \beta, z+\delta)+4 G(x, \beta, z)) / 6 & \text { if } x \in[-0.8,-0.6] \\ 2 & \text { if } x \in[-0.4,-0.2] \\ 2-|10(x-0.1)| & \text { if } x \in[0.0,0.2] \\ 1+(F(x, \alpha, a-\delta)+F(x, \alpha, a+\delta)+4 F(x, \alpha, a)) / 6 & \text { if } x \in[0.4,0.6] \\ 1 & \text { else }\end{cases}
$$

with parameter $a=0.5, z=-0.7, \delta=0.005, \alpha=10, \beta=\log 2 /\left(36 \delta^{2}\right)$ and functions $F, G$ defined as $G(x, \beta, z)=\exp \left(-\beta(x-z)^{2}\right)$, and $F(x, \alpha, a)=\sqrt{\max \left(1-\alpha^{2}(x-a)^{2}, 0\right)}$. The analytic profile is shown in figure 4 by the black solid line. We would like to remark that the cyclic remapping test case is not equivalent to the classical Eulerian test case for which these shapes are traveling with a constant speed across 


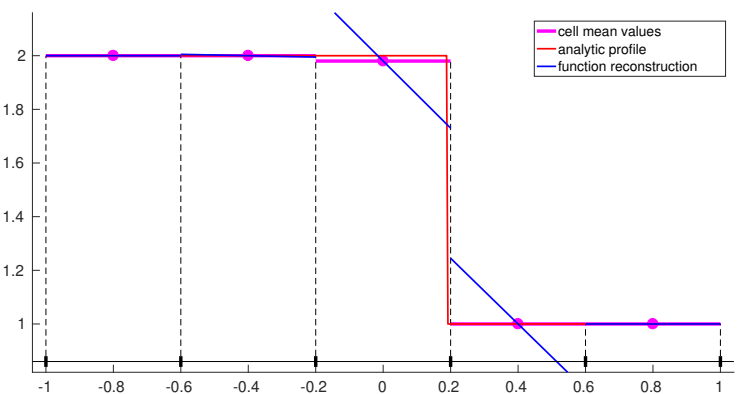

(a) $\mathbb{P}_{1}$

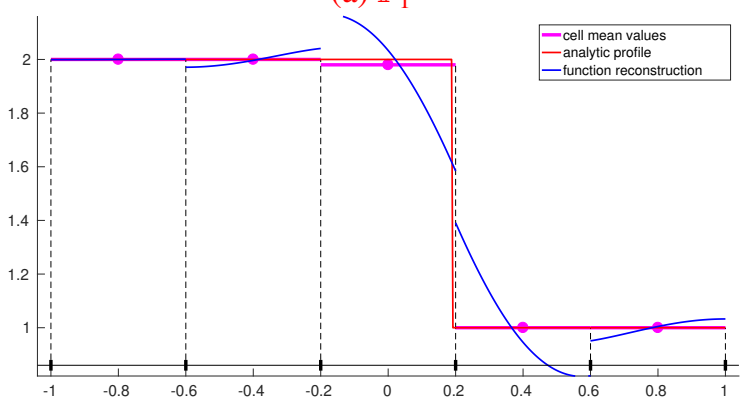

(c) $\mathbb{P}_{4}$

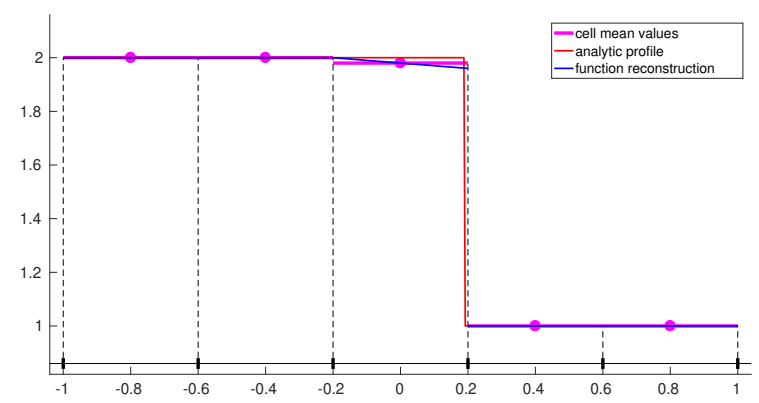

(b) $\mathbb{P}_{1}^{\text {lim }}$

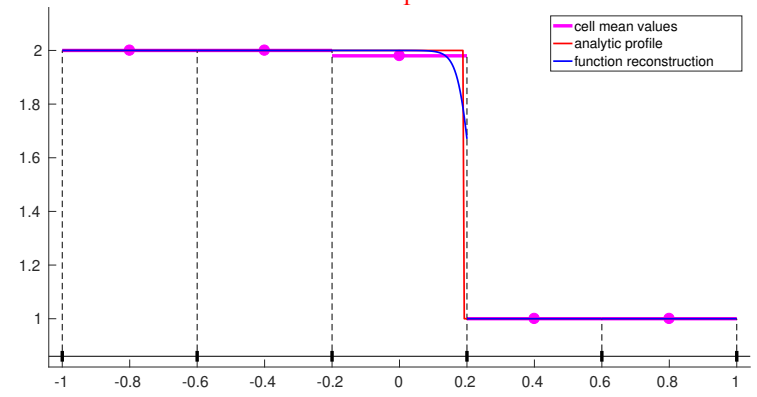

(d) THINC

Figure 3: Static reconstruction problem of a step like function at position $x_{d}=0.19$. Different reconstructions are shown: $\mathbb{P}_{1}$ (a), $\mathbb{P}_{1}^{\lim }$ (b), $\mathbb{P}_{4}$ (c) and THINC (d). The last panel presents THINC reconstruction in the central cell and left/right shifted stencils for $\mathbb{P}_{4}$ reconstructions for the second/fourth cells respectively.

a uniform fixed mesh under periodic boundary conditions. Contrarily, here, being a remapping problem, one accumulates the diffusion errors while the mesh is compressing and expanding non-uniformly. Indeed, in the case of a cyclic remap with $I=128$ cells, the number of cells covering for instance the Gaussian shape is initially 13 , then reduces to 8 and increases up to 21 several times.

We simulate the problem with $I=41,81,161,321,641,1281$ and 2561 cells. The odd number of cells is used in order to avoid boundaries of the shapes to be aligned with the mesh nodes. The positions of the jumps are $x=-0.4$ and $x=-0.2$ and the cells into which they belong are treated with the THINC reconstruction. The cells containing any other discontinuities of the profile, i.e. at locations $x=0,0.1,0.2$, 0.4 and 0.6 , do employ a $\mathbb{P}_{1}^{\lim }$ reconstruction. The remaining cells are treated with $\mathbb{P}_{4}$ possibly with a shifted stencil to avoid the reconstruction neighborhood to "see" discontinuity.

In figure 4, we present the results obtained with $I=41,81,161$ and 321 cells. The results show, that the remap schemes using unlimited polynomial reconstructions suffer from Gibbs like phenomenon (red and blue symbols). However, the $\mathbb{P}_{4}$ results are truly accurate in all smooth areas, especially for regular extrema and shapes. The use of a slope limiter with piece-wise linear reconstruction dissipates the oscillations, and the results are maintained in bounds to the price of excessive diffusion (green symbols) especially on smooth extrema and jumps. The use of THINC reconstructions supplemented with $\mathbb{P}_{1}^{\text {lim }}$ and $\mathbb{P}_{4}$ (with shifted stencil) is genuinely improving the quality of the results. There are no spurious oscillations. The THINC reconstruction captures the jump inside one unique cell, and the $\mathbb{P}_{4}$ reconstructions maintain high accuracy in smooth areas. The $\mathbb{P}_{1}^{\text {lim }}$ reconstruction also ensures that there is no undershoot, and in most regions, the results almost coincide with the unlimited $\mathbb{P}_{4}$ ones (blue). With only 41 cells the resolution is extremely low and spurious effects can be seen, but starting from 81 cells and more, the quality of the remap solution improves to be close to perfection with $I \geq 161$. In figure 5, we present the zooms for four different areas (for $I=641$ cells) to enhance the behavior of each remapping strategy. Next in table 1 , we present the 


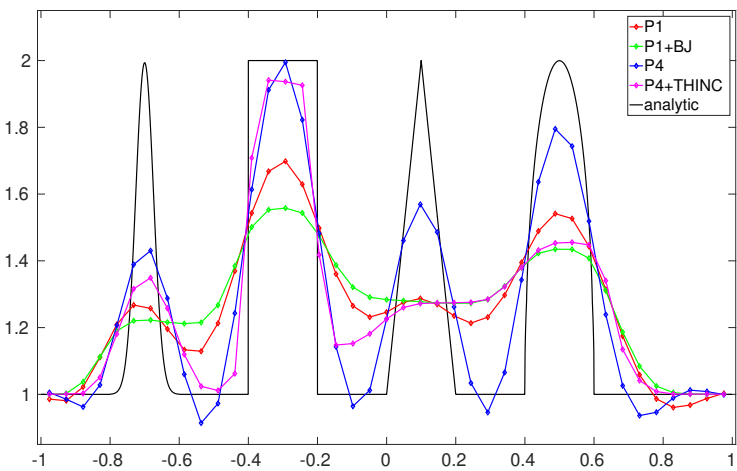

(a) $I=41$

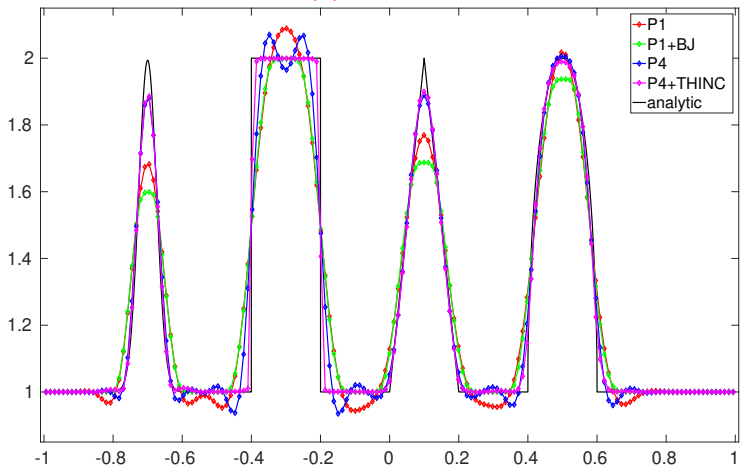

(c) $I=161$

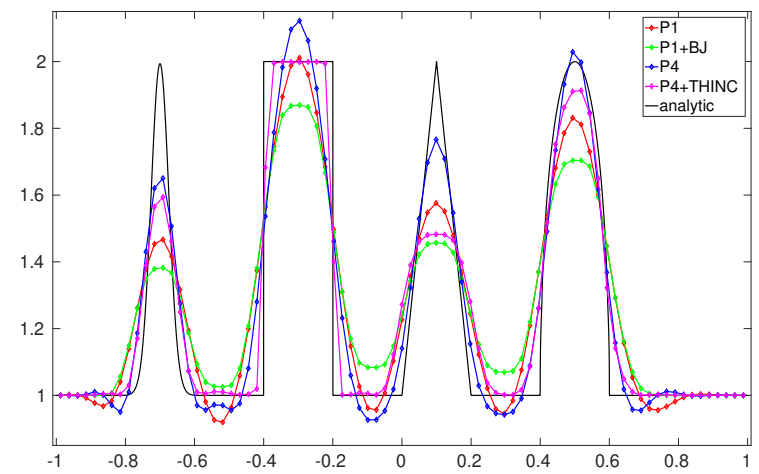

(b) $I=81$

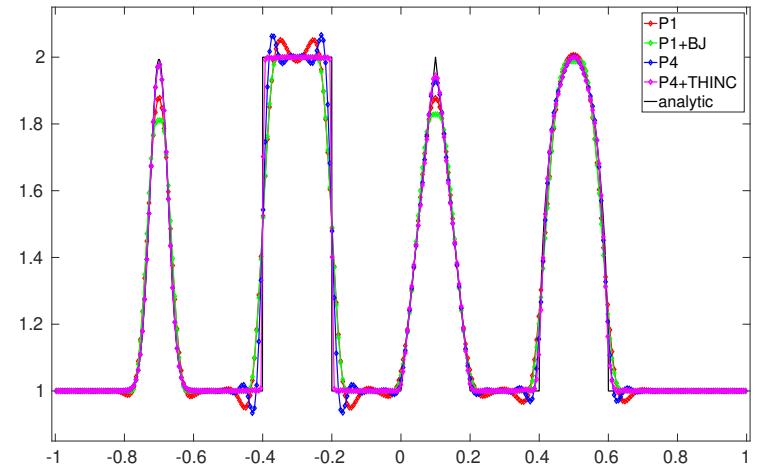

(d) $I=321$

Figure 4: Cyclic remapping of the four profiles with different numbers of cells: 41 (a), 81 (b), 161 (c) and 321 (d). Different remapping schemes shown: unlimited $\mathbb{P}_{1}$ (red), unlimited $\mathbb{P}_{4}$ (blue), $\mathbb{P}_{1}^{\text {lim }}$ (green) and $\mathbb{P}_{4}$ supplemented with THINC (purple).

$L_{1}$ errors computed for the four remapping strategies. We can observe that the unlimited $\mathbb{P}_{4}$ results are more accurate than the $\mathbb{P}_{1}^{\text {lim }}$ even if oscillations are present. More importantly, our strategy with mixed reconstruction is able to reduce the error by a factor 10 compared to the most often used reconstruction $\left(\mathbb{P}_{1}^{\lim }\right)$. It also outperforms the unlimited $\mathbb{P}_{4}$ errors by a factor greater than 5 . The $L_{2}$ errors lead to the same conclusion, therefore we omit those results.

In this numerical section we have observed that a mix of high order polynomials and limited second order ones supplemented with THINC reconstructions for jumps drastically improves the results for static cyclic remapping. These mixed reconstructions are able to associate the accuracy of $\mathbb{P}_{4}$ with the essentially non-oscillatory behavior of $\mathbb{P}_{1}^{\text {lim }}$ and the perfect discontinuity capturing property of THINC. However, to obtain these results, we had to analytically track the jump positions along with the irregularity in the profiles. This discontinuity tracking is feasible for this simple problem, however, this strategy is obviously not viable for the Euler equations. Therefore, in the next section, we supplement the mixed reconstructions with the interface detector described in section 4.3.

\section{Numerical experiments for the full hydrodynamics equations}

In this section, we solve the Euler equations supplemented with a perfect gas equation of state with adiabatic constant equal to $\gamma=7 / 5$. We employ the indirect ALE scheme using the cell-centered Lagrangian method coupled with remapping of all the conservative quantities. The CFL number is fixed at 0.25 , otherwise it is noticed. 


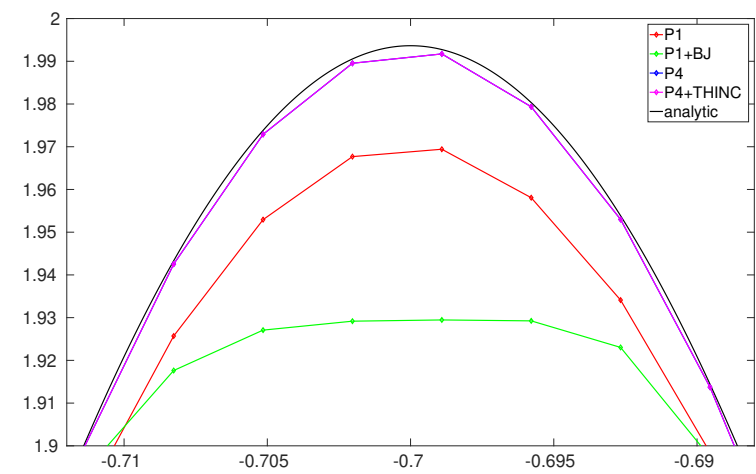

(a)

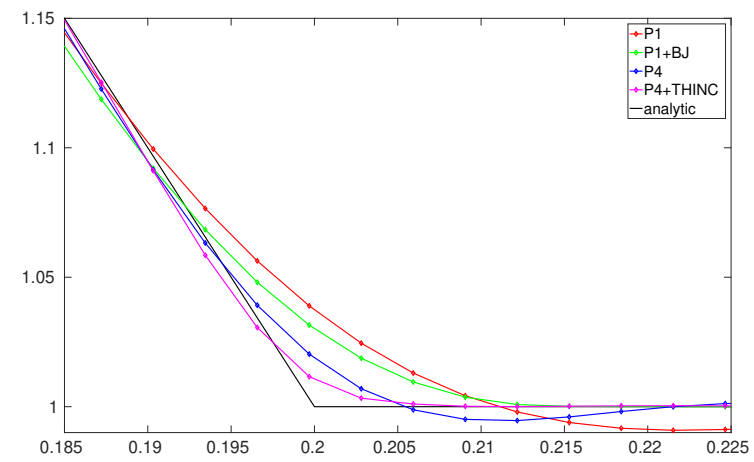

(c)

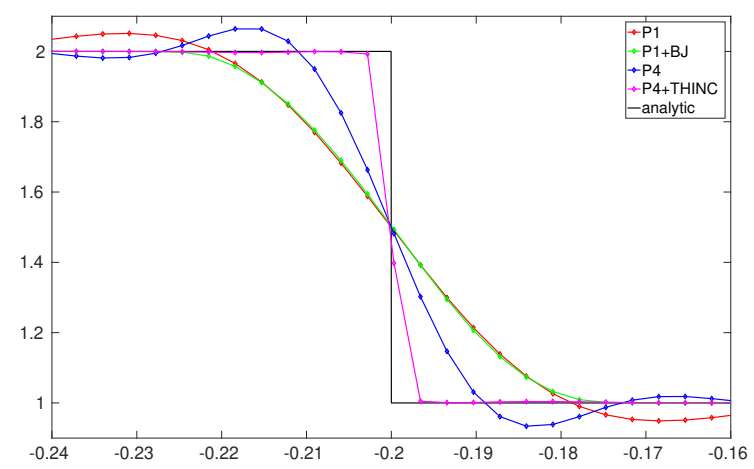

(b)

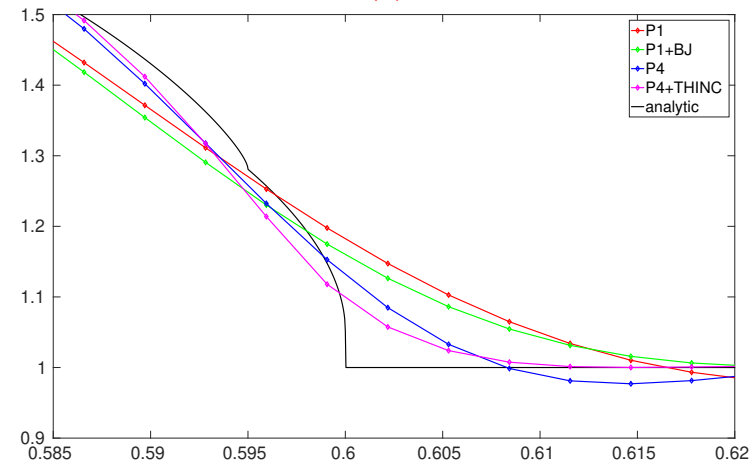

(d)

Figure 5: Cyclic remapping of the four profiles: zooms on four interesting areas are shown for 641 cells. Different remapping schemes shown: unlimited $\mathbb{P}_{1}$ (red), unlimited $\mathbb{P}_{4}$ (blue), $\mathbb{P}_{1}^{\text {lim }}$ (green) and $\mathbb{P}_{4}$ supplemented with THINC (purple).

We ought to compare the behavior of different remapping strategy implemented within the same framework, namely:

LAG only uses the cell-centered Lagrangian scheme.

ALE: P0 uses the low order accurate remap (donor cell remap).

ALE: P1 employs unlimited $\mathbb{P}_{1}$ reconstructions without limiting strategy.

ALE: P1+BJ employs $\mathbb{P}_{1}$ reconstruction with Barth-Jespersen slope limiter, and this is the classical ALE scheme.

ALE: $\mathbf{P 4}$ uses unlimited $\mathbb{P}_{4}$ reconstructions.

ALE: P4+THINC uses the mix of $\mathbb{P}_{4}, \mathbb{P}_{1}^{\text {lim }}$, and THINC reconstructions where appropriate.

We plan to verify numerically that high accurate remapping schemes do generate precise and robust numerical solutions. Notice, that we can not expect to reach a measurable high-order of accuracy with our ALE scheme because the Lagrangian engine is at most a second-order accurate one. Moreover, we are mostly interested in solving problems involving shock waves and discontinuities, for which the order of convergence can not exceed one. However, for a given mesh, some remapping strategy can produce better accurate results.

The rezone strategy considers the original mesh as the rezone one for most tests, leading to an Eulerian 


\begin{tabular}{|c|c|c|c|ccc|}
\hline \multirow{2}{*}{$I$} & \multicolumn{5}{|c|}{ Reconstructions used in the remapper } \\
\cline { 2 - 7 } & $\mathbb{P}_{1}$ & $\mathbb{P}_{1}^{l i m}$ & $\mathbb{P}_{4}$ & $\mathbb{P}_{4}+$ THINC & Ratio vs $\mathbb{P}_{1}^{l \text { lim }}$ & Ratio vs $\mathbb{P}_{4}$ \\
\hline 41 & $1.61 \mathrm{e}-1$ & $1.86 \mathrm{e}-1$ & $7.83 \mathrm{e}-2$ & $1.23 \mathrm{e}-1$ & 1.5 & 0.6 \\
81 & $8.36 \mathrm{e}-2$ & $1.01 \mathrm{e}-1$ & $5.21 \mathrm{e}-2$ & $4.25 \mathrm{e}-2$ & 2.4 & 1.2 \\
161 & $5.22 \mathrm{e}-2$ & $4.41 \mathrm{e}-2$ & $2.39 \mathrm{e}-2$ & $7.58 \mathrm{e}-3$ & 5.8 & 3.2 \\
321 & $2.41 \mathrm{e}-2$ & $2.13 \mathrm{e}-2$ & $1.11 \mathrm{e}-2$ & $2.59 \mathrm{e}-3$ & 8.2 & 4.3 \\
641 & $1.21 \mathrm{e}-2$ & $1.05 \mathrm{e}-2$ & $5.73 \mathrm{e}-3$ & $1.05 \mathrm{e}-3$ & 10.0 & 5.4 \\
1281 & $6.32 \mathrm{e}-3$ & $5.34 \mathrm{e}-3$ & $3.00 \mathrm{e}-3$ & $4.65 \mathrm{e}-4$ & 11.5 & 6.5 \\
2561 & $3.50 \mathrm{e}-3$ & $2.92 \mathrm{e}-3$ & $1.65 \mathrm{e}-3$ & $2.33 \mathrm{e}-4$ & 12.5 & 7.1 \\
\hline
\end{tabular}

Table 1: $L_{1}$ errors for the cyclic remapping problem for the remapping strategies corresponding to the numerical results depicted in figure 4 .

regime as Lagrange plus Remap. For the Shu-Osher test, the domain boundary is moving, so mesh smoothing by simple averaging is used as rezoning.

We first consider some ultra classical test cases involving simple waves, Sod [75] and Lax [76] shock tube problems. Then, more complex interacting wave problems, such as the Collela-Woodward blastwave [77] and Shu-Osher test [78] are simulated. An overview of the initial data is summarized in table 2. Successively refined meshes are considered made of $I=100 \mathrm{~K}$ cells with $K=1,2,4,8$ to measure and observe the mesh convergence of the ALE schemes. Moreover, we systematically present space/time figures, where the cell reconstruction types (THINC for contact and $\mathbb{P}_{1}^{\text {lim }}$ for shocked and unsafe area) are plotted.

\begin{tabular}{|c|c|c|c|c|c|c|c|c|c|c|c|c|}
\hline \multirow[t]{2}{*}{ Test } & \multicolumn{5}{|c|}{ Left state } & \multicolumn{5}{|c|}{ Right state } & \multirow{2}{*}{$\begin{array}{l}\text { Interface } \\
\text { in domain }\end{array}$} & \multirow{2}{*}{$\begin{array}{c}\text { Time } \\
t_{\text {final }}\end{array}$} \\
\hline & \multicolumn{2}{|l|}{$\rho$} & $u$ & \multicolumn{2}{|c|}{$p$} & \multicolumn{3}{|c|}{$\rho$} & $u$ & $p$ & & \\
\hline Sod & \multicolumn{2}{|c|}{1.0} & 0. & \multicolumn{2}{|c|}{1.0} & \multicolumn{3}{|c|}{0.125} & 0.0 & 0.1 & $x_{0}=0.5 \in[0: 1]$ & 0.2 \\
\hline Lax & \multicolumn{2}{|c|}{1.0} & 0. & \multicolumn{2}{|c|}{1000.0} & \multicolumn{3}{|c|}{1.0} & 0.0 & 0.01 & $x_{0}=0.5 \in[0: 1]$ & 0.012 \\
\hline Shu-Osher & \multicolumn{2}{|c|}{3.857143} & 2.629 & \multicolumn{2}{|c|}{10.33333} & $1+$ & $2 \sin (5$ & $\pi x)$ & 0 & 1 & $x_{0}=-4 \in[-9.5: 4.5]$ & 0.18 \\
\hline \multirow{2}{*}{\multicolumn{2}{|c|}{ Test }} & \multicolumn{3}{|c|}{ Left state } & \multicolumn{3}{|c|}{ Middle state } & \multicolumn{3}{|c|}{ Right state } & Interface & Time \\
\hline & & $\rho$ & $u$ & $p$ & $\rho$ & $u$ & $p$ & $\rho$ & $u$ & $p$ & in domain $\Omega=[0: 1]$ & $t_{\text {final }}$ \\
\hline Collela-Woc & dward & 1.0 & 0.0 & 1000.0 & 1.0 & 0.0 & 0.01 & 1.0 & 0.0 & 100 & $x_{0}=0.1, x_{1}=0.9$ & 0.038 \\
\hline
\end{tabular}

Table 2: Initial conditions for the 1D Riemann problems and other tests simulated in this paper.

\subsection{Sod test case}

The Sod problem is a 1D Riemann shock tube with a mild discontinuity [75] generating simple waves. The exact solution consists in a left moving rarefaction fan, a right moving contact discontinuity and a shock wave. The domain $\Omega=[0 ; 1]$ is filled with an ideal gas at rest with adiabatic constant $\gamma=1.4$. Two states are separated by a discontinuity initially located at $x=0.5$. The density/pressure on the left side of the discontinuity are $(\rho, p)=(1.0,1.0)$ and $(0.125,0.1)$ on the right side, see table 2 . The final time is set to $t_{\text {final }}=0.2$ and wall type boundary conditions are set. In figure 6 , we present the mesh convergence study for $I=100,200,400$ and 800 cells for the Lagrangian scheme and ALE with remap strategy P1, P1+BJ, P4 and P4+THINC. In figures 7 and 8, we present the density profiles only and zooms at contact and shock wave. The velocity, pressure and specific internal energy profiles for 100 and 400 cells are presented in figure 9 . Recall, that the exact position of the contact discontinuity is $x_{C} \simeq 0.6855$ while the shock is located at $x_{S} \simeq 0.8504$. We omit the results produced by $\mathbb{P}_{0}$ reconstruction because they are overly diffusive and they do not bring any useful information here. The numerical method using mixed reconstruction produces systematically better results in terms of accuracy and non-oscillatory behavior. The sharpness of the contact is equivalent to the Lagrangian results, while the shock is free from spurious oscillations and its quality is 
equivalent to $\mathrm{P} 1+\mathrm{BJ}$ or $\mathrm{P} 4$. As expected, using $\mathrm{P} 4$ remapping for every cell generates spurious oscillations, though small enough to avoid a code crashing for this mild shock tube.

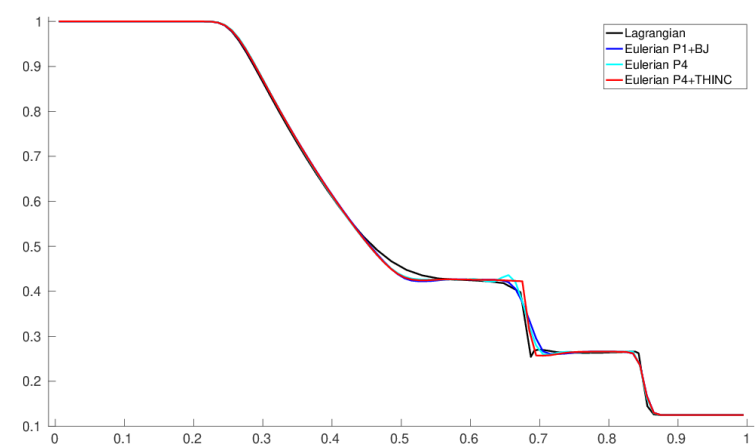

(a) $I=100$

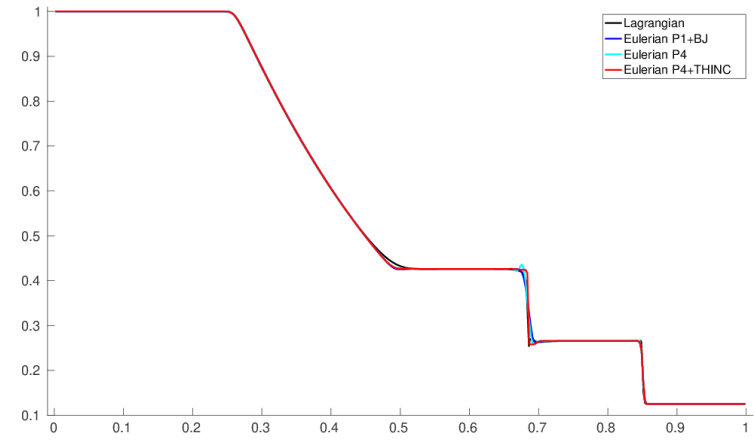

(c) $I=400$

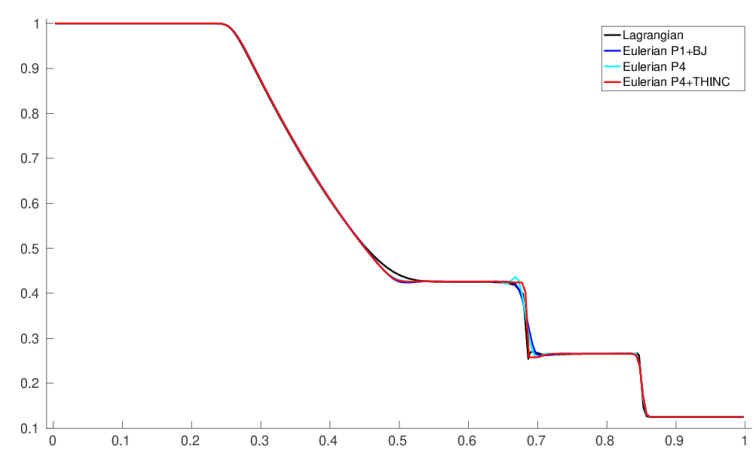

(b) $I=200$

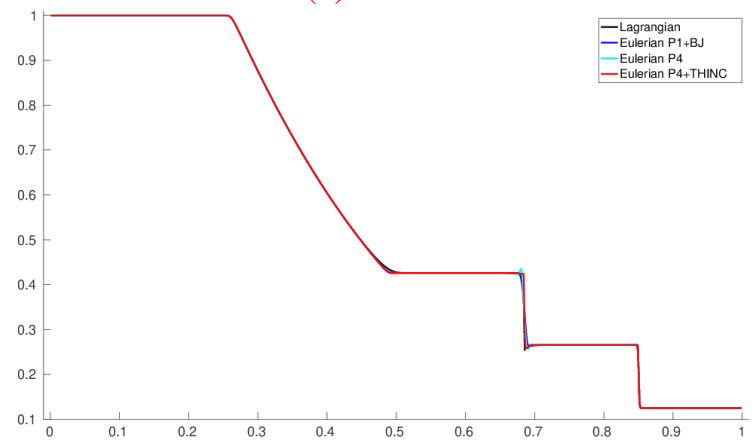

(d) $I=800$

Figure 6: Sod test case: fluid density shown for simulations on 100 (a), 200 (b), 400 (c) and 800 (d) cells.

In table 3, we have gathered the $L_{1}, L_{2}$ and $L_{\infty}$ errors and their associated rates of convergence for the ALE scheme with $\mathbb{P}_{0}, \mathbb{P}_{1}^{\text {lim }}, \mathbb{P}_{4}$ and mixed $\left(\mathbb{P}_{4}+\right.$ THINC) reconstruction types. The analytic solution is obtained from the analytic Riemann solver described in [76], and the numerical errors are computed in the integral sense by the same quadrature rule (11). As expected, the rates of convergence are about 1 for any high accurate remap strategy, with the best errors obtained by the mixed reconstructions. In figure 10, we plot in log-scale the data from table 3. To illustrate these results, the needed mesh size to reach an $L_{2}$ error of about $10^{-4}$ is about 159 cells for P4+THINC, 244 for $\mathrm{P} 4,292$ for $\mathrm{P} 1+\mathrm{BJ}$, more than 2000 for P0, and 223 for the Lagrangian scheme. It means that the gain in terms of mesh size is of the order 1.8 between our mixed reconstruction approach and the classical second-order + limiter remap strategy. The (c) panel in figure 10 demonstrates that the discrepancy magnitude is lowest for the new method for all resolutions, although no convergence in $L_{\infty}$ norm can be expected due to discontinuous solution.

At last, we present the cell reconstruction type for all cells and all time-steps in figure 11. The cell type is determined by the interface detector. We mark the THINC cells with a red symbol, the P1+BJ cells with a blue one, and nothing (white symbol) is used for the P4 cells. The time-steps are in $y$-direction while the abscissa refers to the cell position. We observe that the interface detector is able to track the contact discontinuity (surrounded by two P1+BJ cells) and, later the shock wave when it escapes the contact zone.

The Sod shock tube has been used as a sanity check because its relatively weak discontinuity renders this test easy to simulate. We have observed however that using mixed reconstruction along with the interface detector drastically improves the resolution of the contact discontinuity: it is indeed now captured in a single cell. 


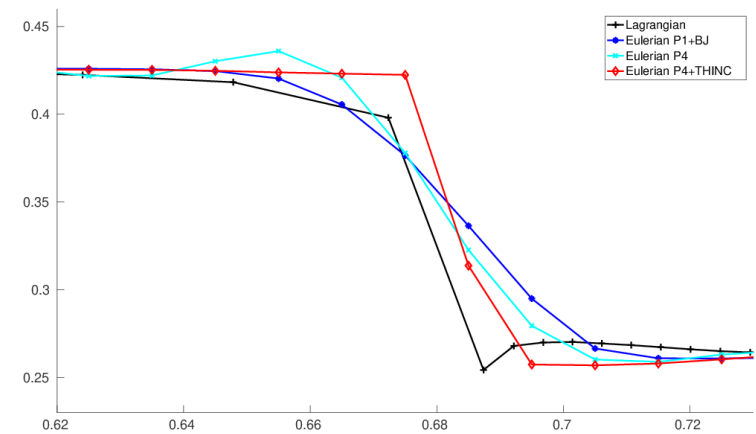

(a) $I=100$

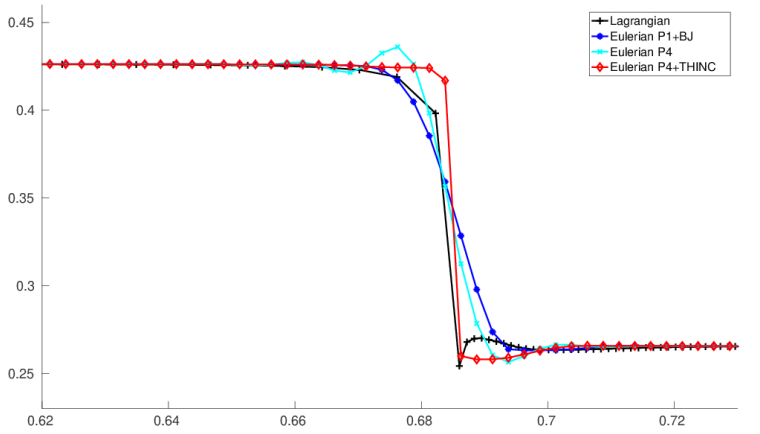

(c) $I=400$

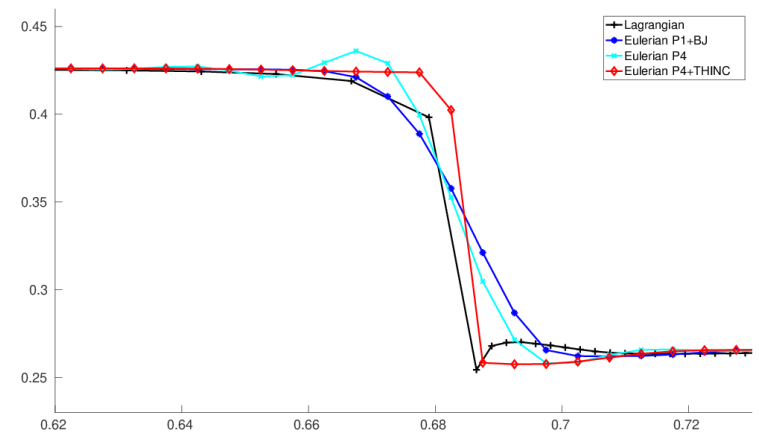

(b) $I=200$

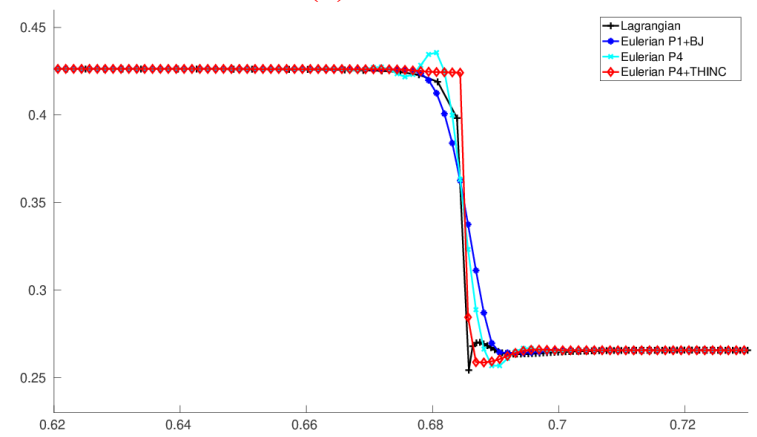

(d) $I=800$

Figure 7: Sod test case: fluid density shown for simulations on 100 (a), 200 (b), 400 (c) and 800 (d) cells - zoom at contact wave.

\subsection{Lax test case}

The Lax problem is a shock tube with more severe contact discontinuity and shock wave than Sod [76]. The initial data are gathered in table 2. In figure 12, we present the mesh convergence study for fluid density. The full view is plotted in the left panels, a zoom at the contact and shock waves in the right panels. As expected the $\mathbb{P}_{4}$ remapper produces oscillatory results while $\mathbb{P}_{1}^{\text {lim }}$ one can eliminate the oscillations for the price of an excessive numerical dissipation especially at the contact wave $(8-10$ cells over which the contact is spread). Contrarily, the mixed reconstruction $\left(\mathbb{P}_{4}, \mathbb{P}_{1}\right.$ lim, THINC) is able to capture the contact inside a single cell, avoiding the numerical oscillations with, however, a spurious effect just after the contact, resulting from the oscillations generated by the Lagrangian scheme. The shock wave is captured with the same accuracy by all schemes, that is inside one or two cells. We did not expect any improvement regarding the shock wave. Figure 13 presents the cell reconstruction types as a function of space and time. Clearly, the contact discontinuity and the shock wave are tracked appropriately.

In figure 14, we present the $L_{1}, L_{2}$ and $L_{\infty}$ error plots produces by all schemes. While the Lagrangian scheme generates the smallest errors, the mixed reconstruction in the remap is the best of the ALE schemes tested here apart from the smallest mesh size. The order of convergence is about the same for all methods, that is less than 0.8. If one sets a required level of $L_{2}$ error at 0.02 then the P1+BJ ALE scheme would employ about 590 cells while the P4+THINC about 300 (320 for P4). The gain in the number of cells is about 2 .

\subsection{Shu-Osher oscillatory test case}

This test [78] is a 1D hydrodynamic shock tube for which the downstream flow has a sinusoidal density fluctuation, see table 2. Due to the fact that the left boundary is a piston, the computational domain shrinks 


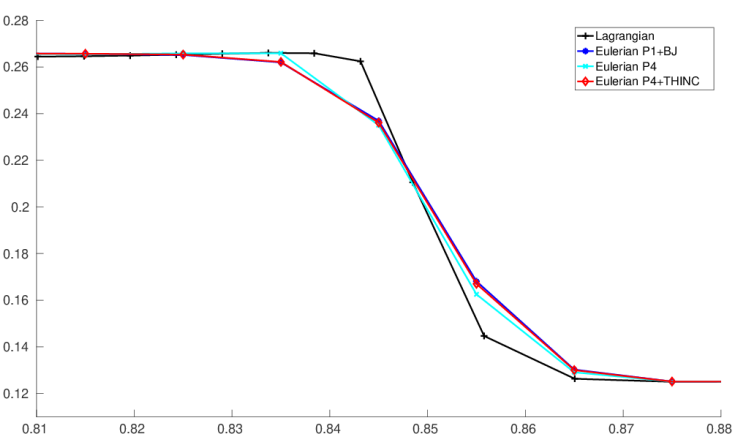

(a) $I=100$

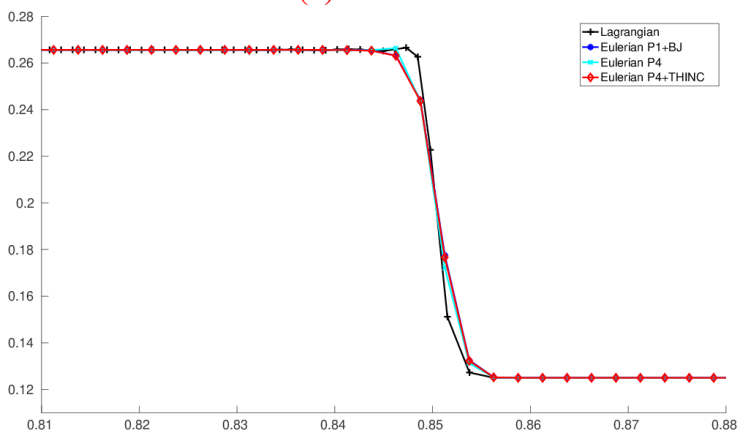

(c) $I=400$

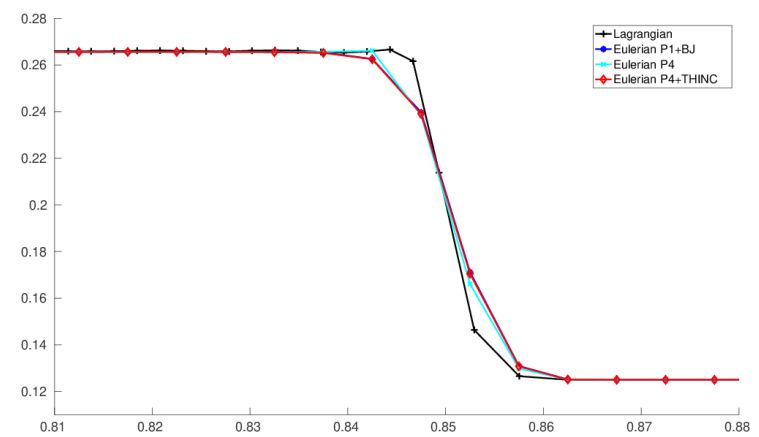

(b) $I=200$

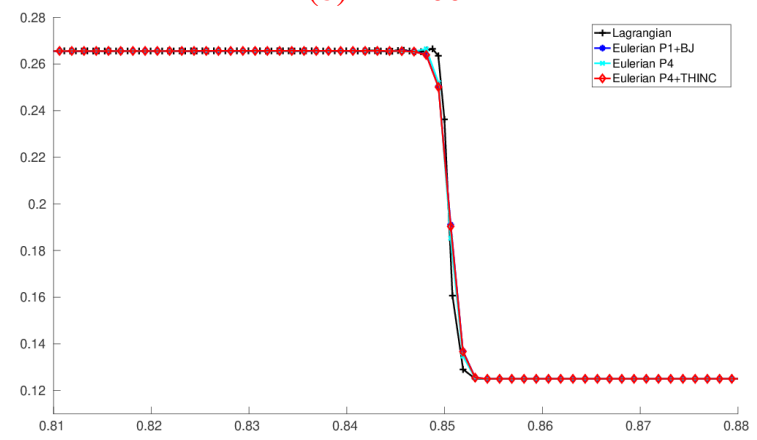

(d) $I=800$

Figure 8: Sod test case: fluid density shown for simulations on 100 (a), 200 (b), 400 (c) and 800 (d) cells - zoom at shock wave.

in time. As a consequence, the rezone strategy does operate a Laplacian smoothing strategy by averaging the nodal positions. As such, the simulations are in a true ALE mode, and no more in a Lagrange plus remap one. Notice that the unlimited $\mathbb{P}_{4}$ remapper is not able to simulate this problem due to oscillations in internal energy leading to time-step degeneration. Moreover, the $\mathbb{P}_{0}$ remapper produces genuinely diffused results and we skip those curves. In figure 15, we present the mesh convergence study for the density variable for the Lagrangian, ALE-P1+BJ and ALE-P4+THINC schemes. A zoom at the oscillatory area is solely proposed. The right panels present the associated cell reconstruction types as detected by our interface tracker. As expected, only a contact and a shock wave are captured in their motion. It seems that the use of $\mathbb{P}_{4}$ reconstructions slightly improves the accuracy of the numerical solution compared to the classical second-order accurate remapper. For this test case, the contact discontinuity seems to fall in the unlikely situation where the surrounded cells do not form a strictly increasing or decreasing set of data for lower-resolution simulations. This implies that the THINC function can not be reconstructed properly, it degenerates to $\mathbb{P}_{0}$, and the mean value in this cell is considered instead. Contrarily, for the 800 cell resolution, the contact zone contains enough cells, the THINC reconstruction can operate appropriately, and one retrieves a sharp contact, mimicking the Lagrangian results.

\subsection{Woodward-Collela blastwave}

The Woodward-Collela blastwave is a classical double shock tube where simple waves do interact [77]. The left, middle, and right states are given in table 2. Two shock waves and two contact discontinuities develop and propagate towards the wall boundary conditions and reflect from them. These initial simple waves further interact creating a more complex flow pattern.

In figure 16, we present the numerical density obtained by the ALE schemes for the same refined meshes as previously. Once again, the unlimited $\mathbb{P}_{4}$ remapper can not simulate this problem. Moreover 


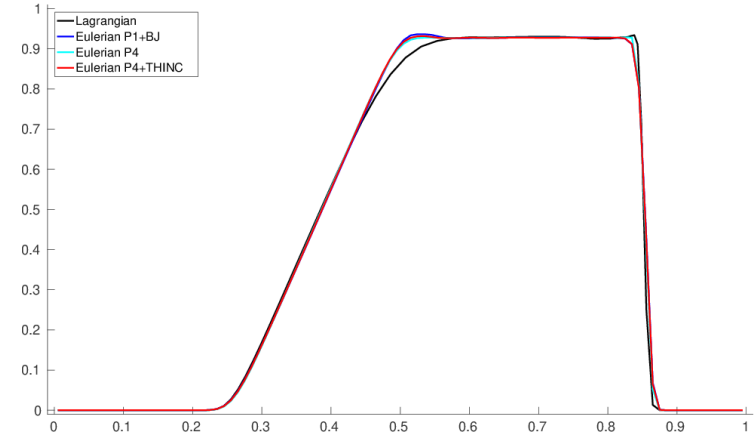

(a) $I=100$, velocity

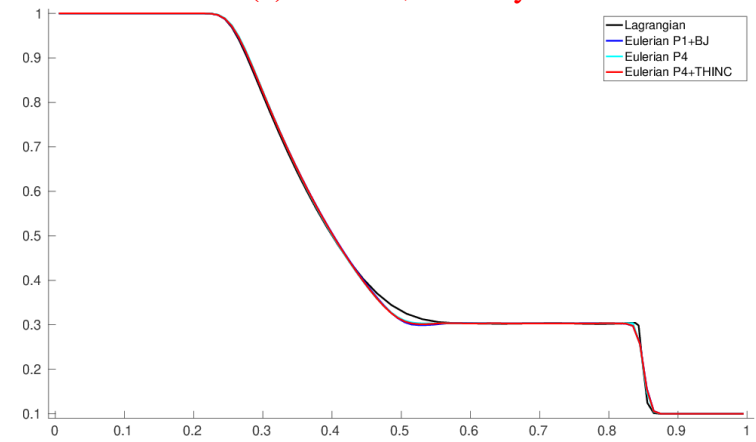

(c) $I=100$, pressure

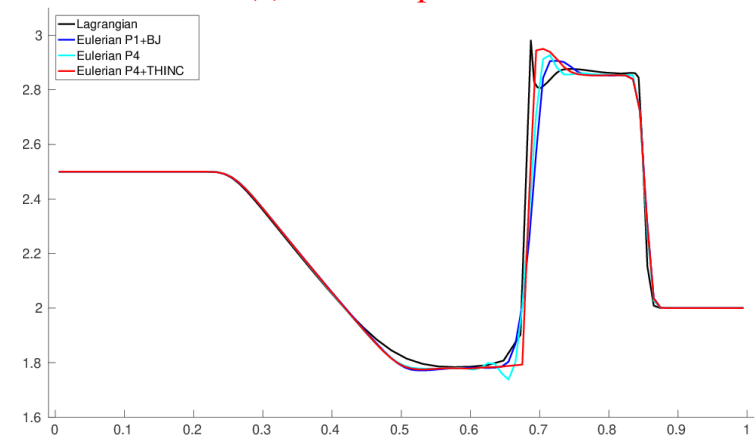

(e) $I=100$, specific internal energy

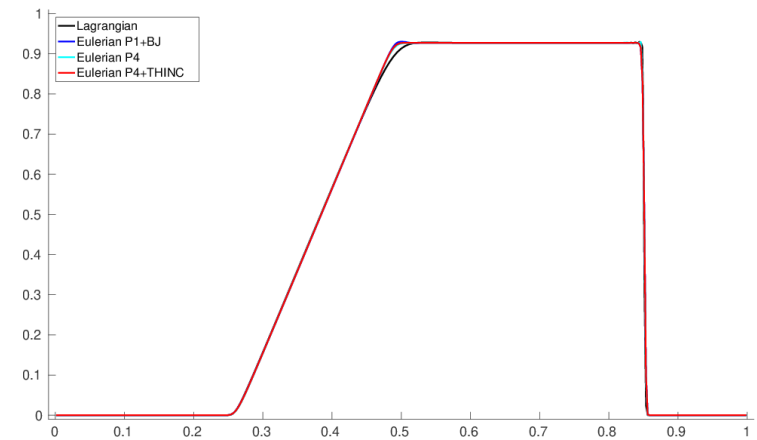

(b) $I=400$, velocity

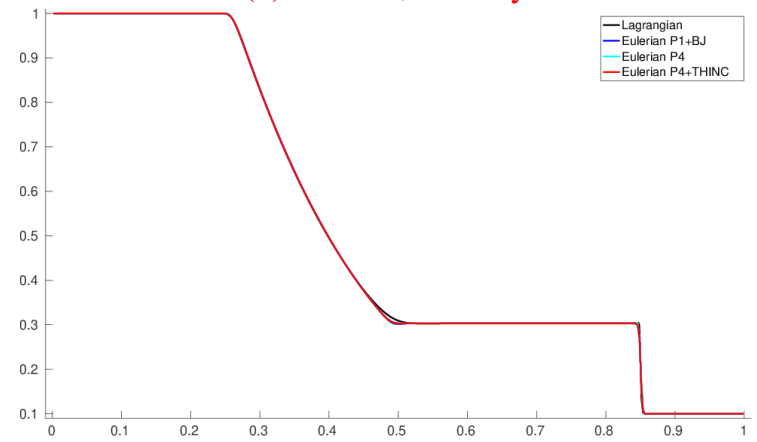

(d) $I=400$, pressure

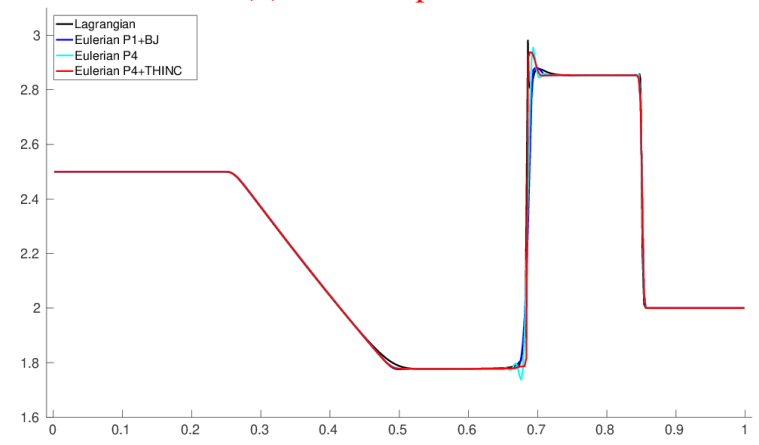

(f) $I=400$, specific internal energy

Figure 9: Sod test case: fluid quantities shown for 100 cells (left panels) and 400 cells (right panels) - velocity (a-b), pressure (c-d), and specific internal energy (e-f). 


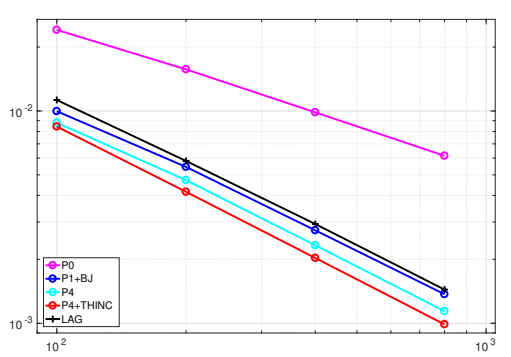

(a) $L_{1}$

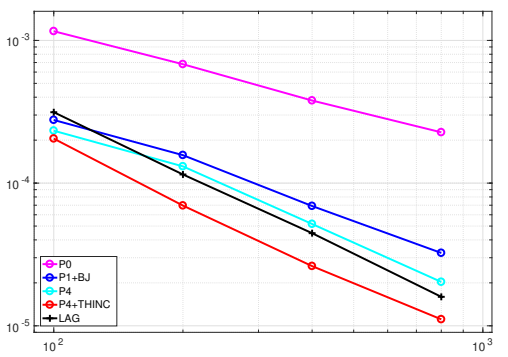

(b) $L_{2}$

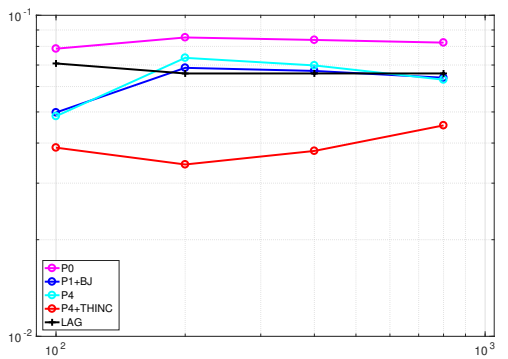

(c) $L_{\infty}$

Figure 10: $L_{1}$ (a), $L_{2}$ (b), and $L_{\infty}$ (c) density errors for Sod problem from table 3, produced by the Lagrangian scheme and the ALE schemes incorporating $\mathbb{P}_{0}, \mathbb{P}_{1}^{\text {lim }}, \mathbb{P}_{4}$, and mixed $\left(\mathbb{P}_{4}+\right.$ THINC) reconstructions.

\begin{tabular}{|c|c|c|c|c|c|c|c|c|c|c|c|c|}
\hline Sod & \multicolumn{6}{|c|}{$\mathbb{P}_{0}$} & \multicolumn{6}{|c|}{$\mathbb{P}_{1}^{\lim }$} \\
\hline $\mathrm{h}$ & $L_{1}$ & $L_{2}$ & $L_{\infty}$ & $R_{1}$ & $R_{2}$ & $R_{\infty}$ & $L_{1}$ & $L_{2}$ & $L_{\infty}$ & $R_{1}$ & $R_{2}$ & $R_{\infty}$ \\
\hline 100 & 2.41E-02 & $1.16 \mathrm{E}-03$ & 7.87E-02 & - & - & - & 9.97E-03 & 2.77E-04 & 4.98E-02 & - & - & - \\
\hline 200 & $1.57 \mathrm{E}-02$ & $6.81 \mathrm{E}-04$ & 8.53E-02 & 0.61 & 0.77 & -0.11 & $5.45 \mathrm{E}-03$ & $1.57 \mathrm{E}-04$ & $6.86 \mathrm{E}-02$ & 0.87 & 0.82 & -0.46 \\
\hline 400 & $9.85 \mathrm{E}-03$ & 3.79E-04 & 8.38E-02 & 0.68 & 0.85 & 0.02 & $2.74 \mathrm{E}-03$ & $6.91 \mathrm{E}-05$ & 6.71E-02 & 0.99 & 1.19 & 0.03 \\
\hline 800 & $6.15 \mathrm{E}-03$ & 2.27E-04 & $8.22 \mathrm{E}-02$ & 0.68 & 0.74 & 0.03 & $1.37 \mathrm{E}-03$ & $3.24 \mathrm{E}-05$ & 6.39E-02 & 1.00 & 1.09 & 0.07 \\
\hline Sod & \multicolumn{6}{|c|}{$\mathbb{P}_{4}$} & \multicolumn{6}{|c|}{$\mathbb{P}_{4}+$ THINC } \\
\hline $\mathrm{h}$ & $L_{1}$ & $L_{2}$ & $L_{\infty}$ & $R_{1}$ & $R_{2}$ & $R_{\infty}$ & $L_{1}$ & $L_{2}$ & $L_{\infty}$ & $R_{1}$ & $R_{2}$ & $R_{\infty}$ \\
\hline 100 & 8.80E-03 & 2.33E-04 & 4.85E-02 & - & - & - & 8.44E-03 & 2.05E-04 & 3.87E-02 & - & 一 & - \\
\hline 200 & $4.72 \mathrm{E}-03$ & $1.31 \mathrm{E}-04$ & 7.37E-02 & 0.90 & 0.83 & -0.61 & 4.16E-03 & $6.96 \mathrm{E}-05$ & 3.43E-02 & 1.02 & 1.56 & 0.17 \\
\hline 400 & 2.33E-03 & 5.17E-05 & $6.98 \mathrm{E}-02$ & 1.02 & 1.34 & 0.08 & 2.03E-03 & $2.62 \mathrm{E}-05$ & 3.78E-02 & 1.04 & 1.41 & -0.14 \\
\hline 800 & $1.14 \mathrm{E}-03$ & 2.03E-05 & 6.30E-02 & 1.03 & 1.35 & 0.15 & $9.88 \mathrm{E}-04$ & $1.11 \mathrm{E}-05$ & 4.54E-02 & 1.03 & 1.24 & -0.26 \\
\hline
\end{tabular}

Table 3: $L_{1}, L_{2}$, and $L_{\infty}$ density errors and their associates convergence rates for Sod problem for the ALE scheme with $\mathbb{P}_{0}, \mathbb{P}_{1}^{\text {lim }}$, $\mathbb{P}_{4}$ and mixed $\left(\mathbb{P}_{4}+\right.$ THINC) reconstruction types.

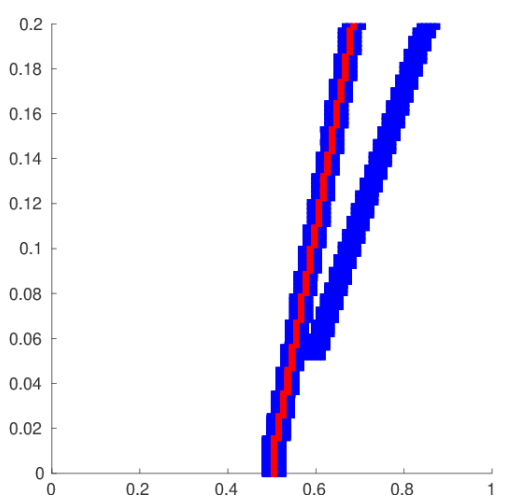

(a) $I=100$

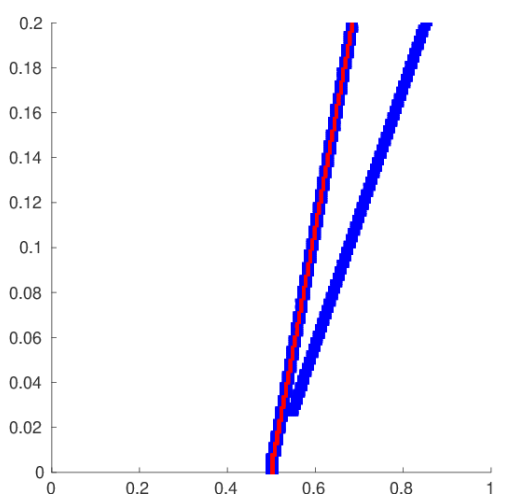

(b) $I=200$

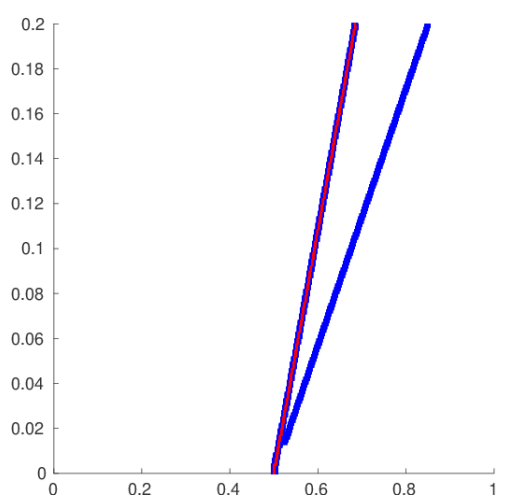

(c) $I=400$

Figure 11: Cell reconstruction type determined by the interface detector as a function of time. Red cells use THINC reconstruction, blue cells use $\mathbb{P}_{1}^{\text {lim }}$ reconstruction, and white cells use $\mathbb{P}_{4}$ reconstruction. Results are shown for 100 (a), 200 (b) and 400 (c) cells. 


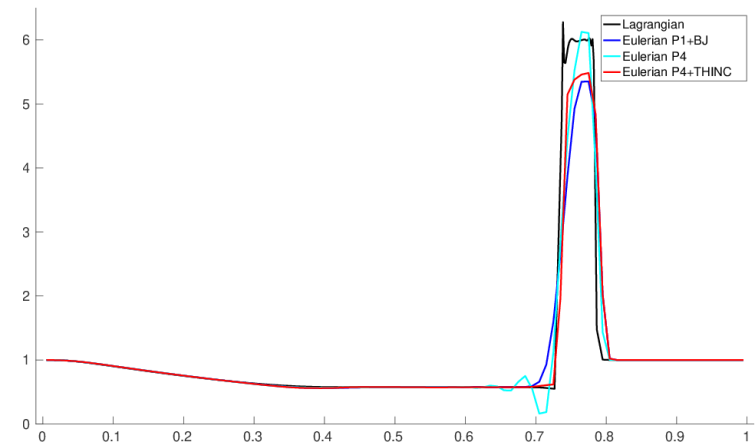

(a) $I=100$

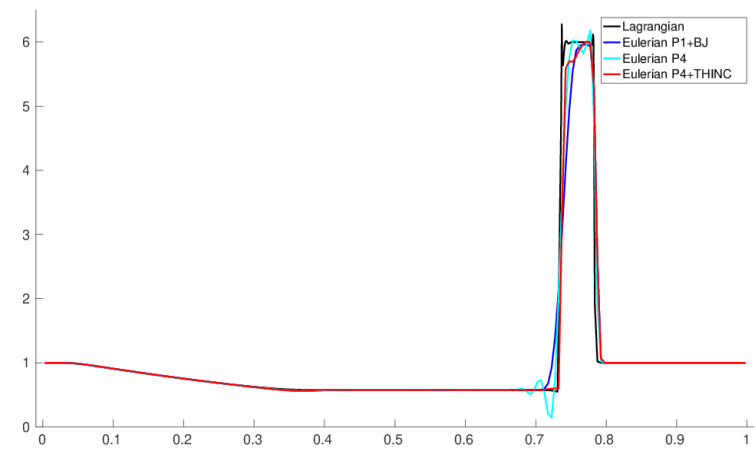

(c) $I=200$

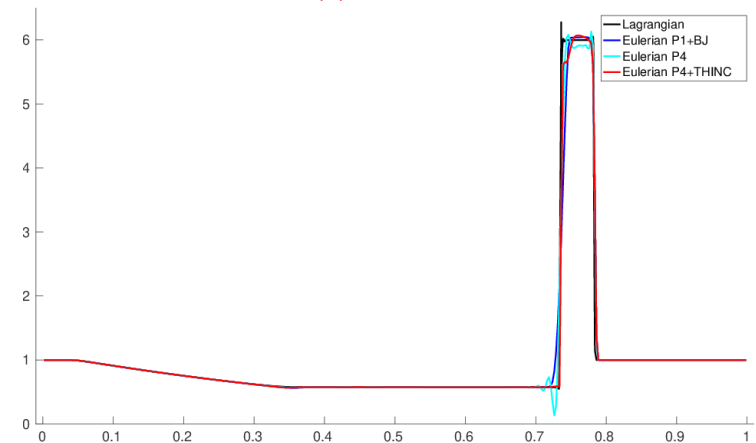

(e) $I=400$

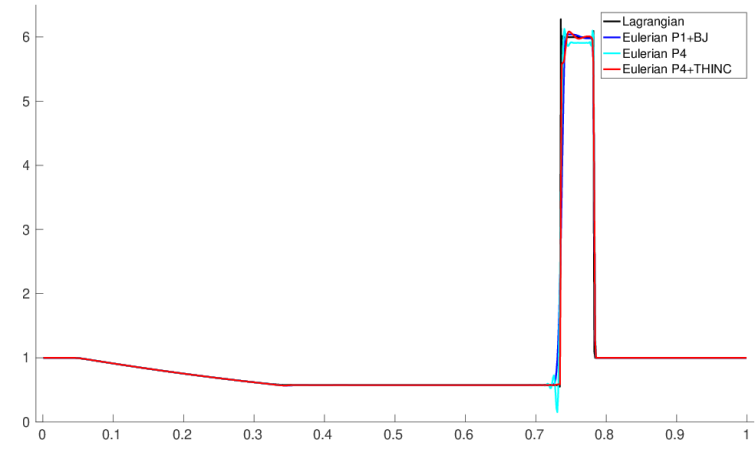

(g) $I=800$

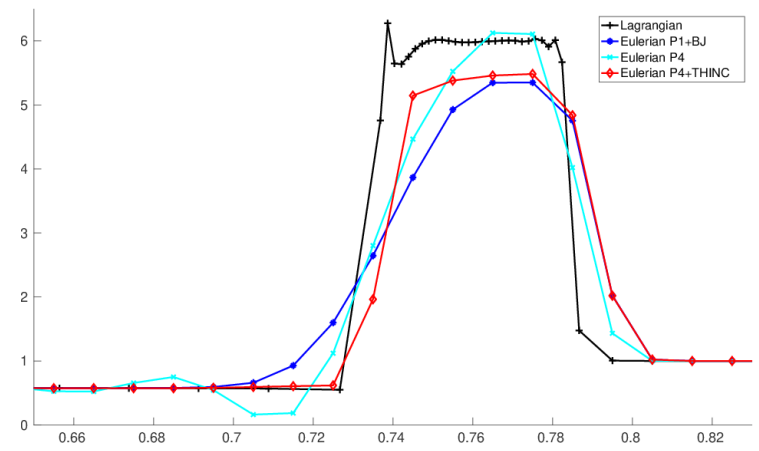

(b) $I=100$, zoom

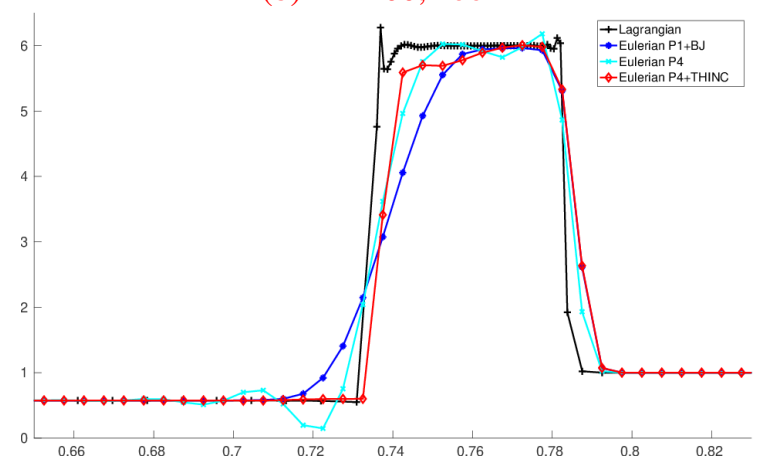

(d) $I=200$, zoom

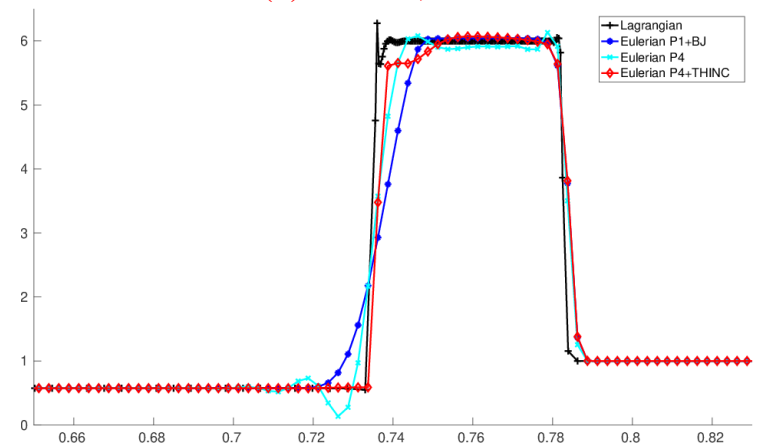

(f) $I=400$, zoom

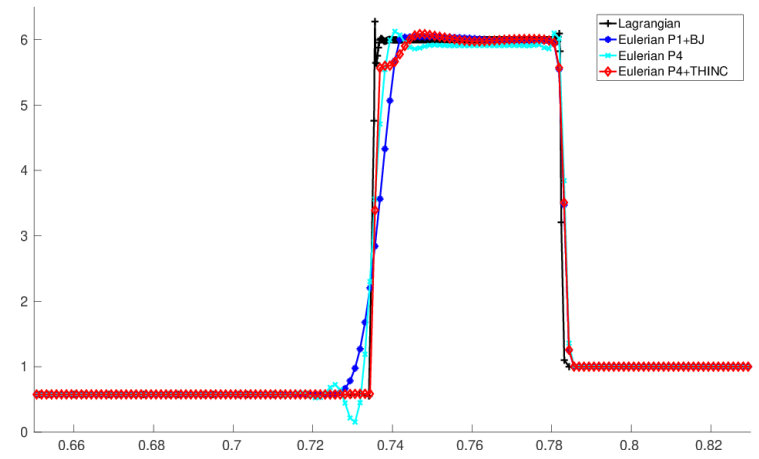

(h) $I=800$, zoom

Figure 12: Lax test case: fluid density shown on 100 (a-b), 200 (c-d), 400 (e-f) and 800 (g-h) cells. Full view (left panels) and zoom on the contact and shock waves (right panels) are shown. 


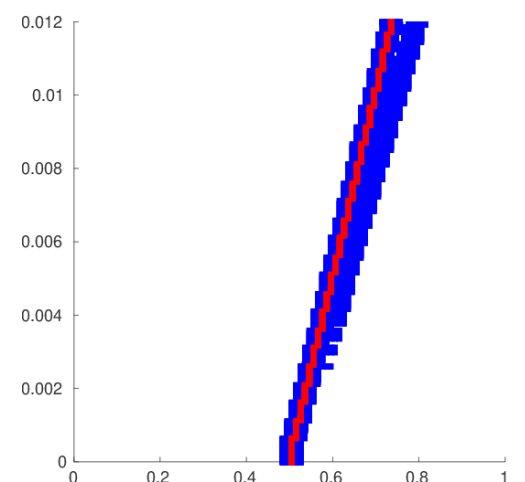

(a) $I=100$

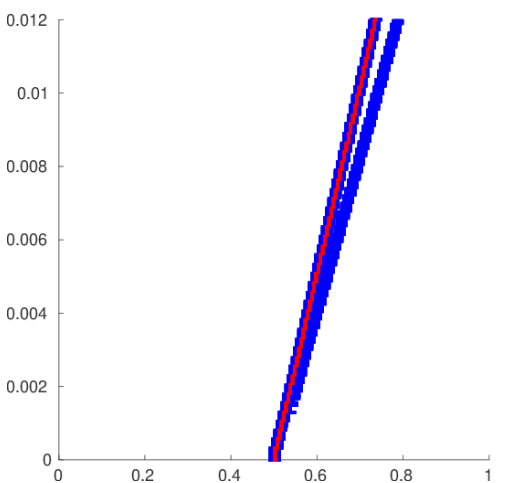

(b) $I=200$

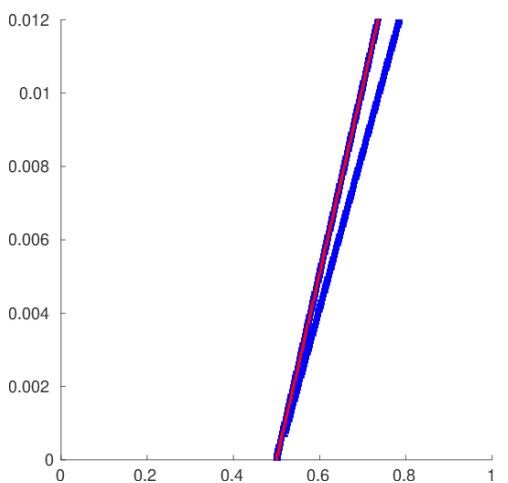

(c) $I=400$

Figure 13: Lax test case: cell reconstruction types as a function of time. Red cells use THINC reconstruction, blue cells use $\mathbb{P}_{1}^{\lim }$ reconstruction, and white cells use $\mathbb{P}_{4}$ reconstruction. Results are shown for 100 (a), 200 (b), and 400 (c), cells.

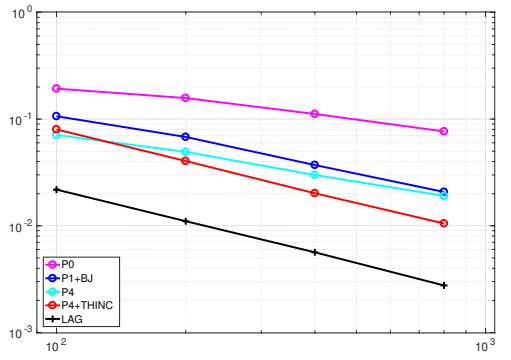

(a) $L_{1}$

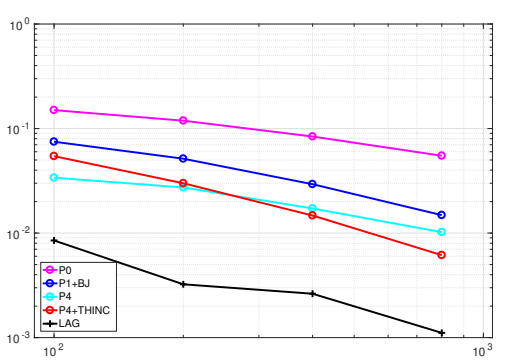

(b) $L_{2}$

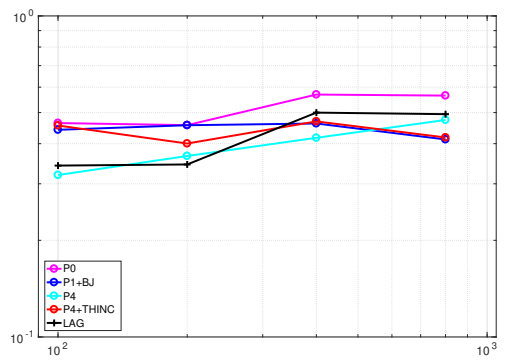

(c) $L_{\infty}$

Figure 14: $L_{1}$ (a), $L_{2}$ (b), and $L_{\infty}$ (c) density errors for Lax problem produced by the Lagrangian scheme and ALE schemes incorporating $\mathbb{P}_{0}, \mathbb{P}_{1}^{\text {lim }}, \mathbb{P}_{4}$, and mixed $\left(\mathbb{P}_{4}+\right.$ THINC) reconstructions.

the $\mathbb{P}_{0}$ remapper produces too diffused results and we skip those curves. Therefore, only the classical $\mathbb{P}_{1}^{\lim }$ and our mixed reconstruction strategy remappers are compared against the Lagrangian scheme solution. It is clear from these figures that the proposed scheme is significantly improving the treatments of the contact discontinuities compared to the classical second-order remapping scheme. Indeed, the contacts are maintained with almost the same quality as in the Lagrangian scheme, that is on one or two cells maximum, while the classical remapper, like any Eulerian scheme, can not do better than tens of cells, especially for the first wave on the left. Few tiny spurious features can still be observed in the left low-density area. The plots of cell reconstruction types (right panels) confirm the fact that the main contact waves and shocks are appropriately tracked by our algorithm. Notice, that the contacts and shocks are clearly interacting with each others. Moreover there are supplementary waves created by those interactions, which are not taken into account by our tracking algorithm as it stands. With a more clever tracking/detecting procedure, we would certainly be able to improve the results even further, for instance close to the contact at location $x \simeq 0.76$, which is not present in the initial data and develops during the interaction of waves, so it is not possible to track it properly with our simple algorithm. 


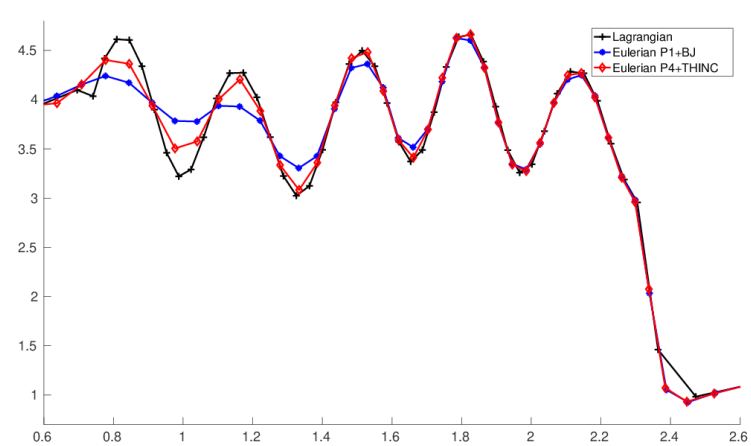

(a) $I=100$, density zoom

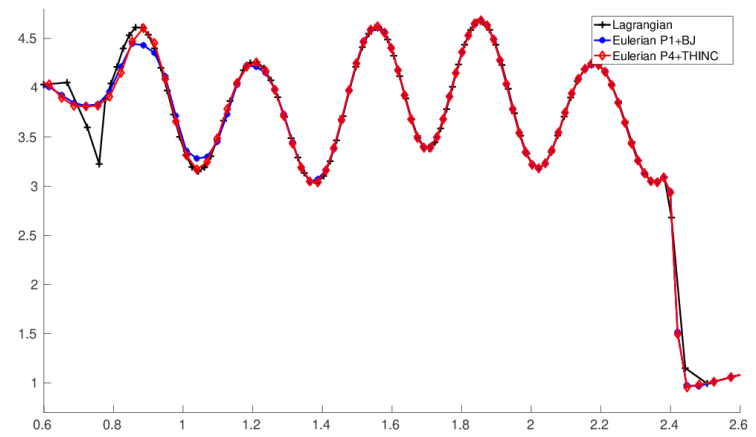

(c) $I=200$, density zoom

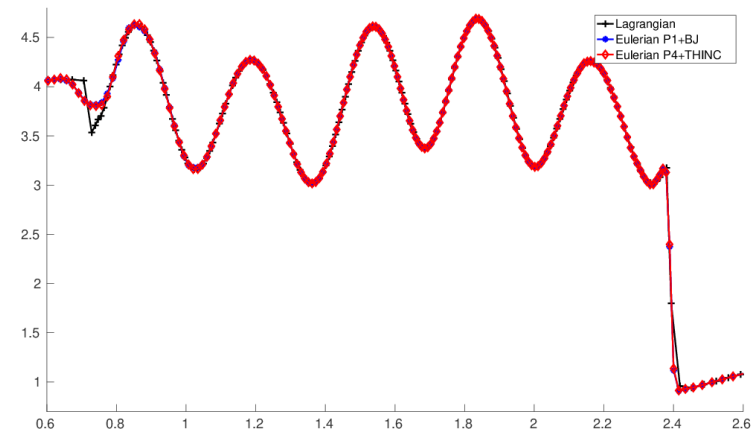

(e) $I=400$, density zoom

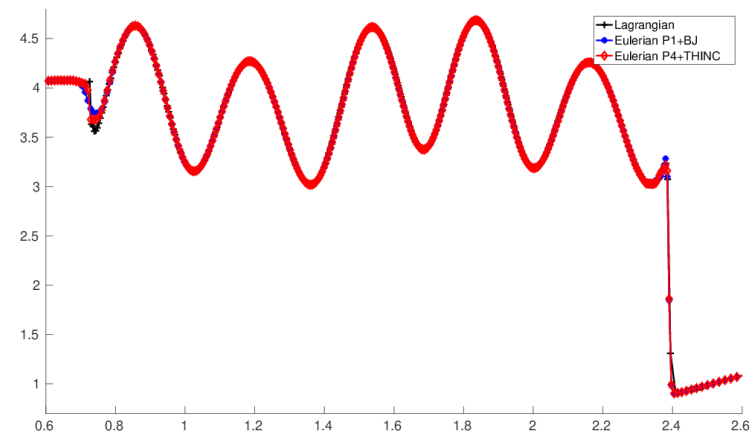

(g) $I=800$, density zoom

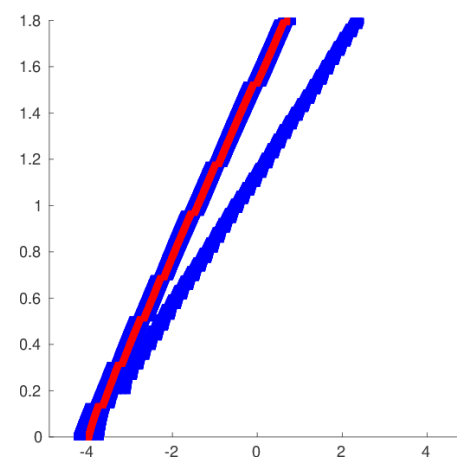

(b) $I=100$, reconstruction type

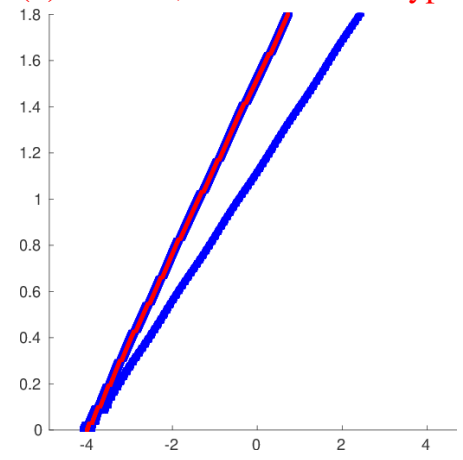

(d) $I=200$, reconstruction type

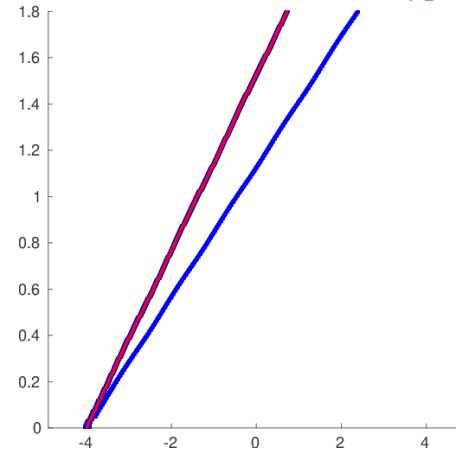

(f) $I=400$, reconstruction type

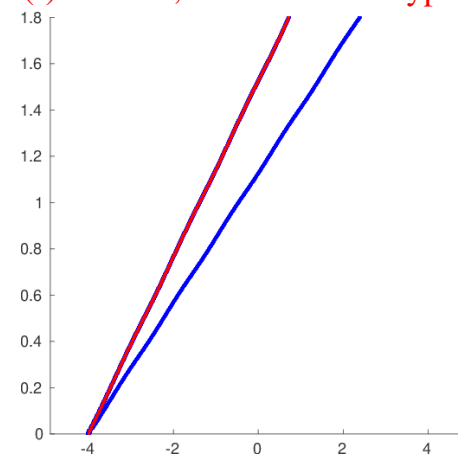

(h) $I=800$, reconstruction type

Figure 15: Shu-Osher oscillatory test case: fluid density shown on 100 (a-b), 200 (c-d), 400 (e-f), and 800 (g-h) cells. Zoom on the central area (left panels) and cell reconstruction types as a function of time (right panels) are shown. 


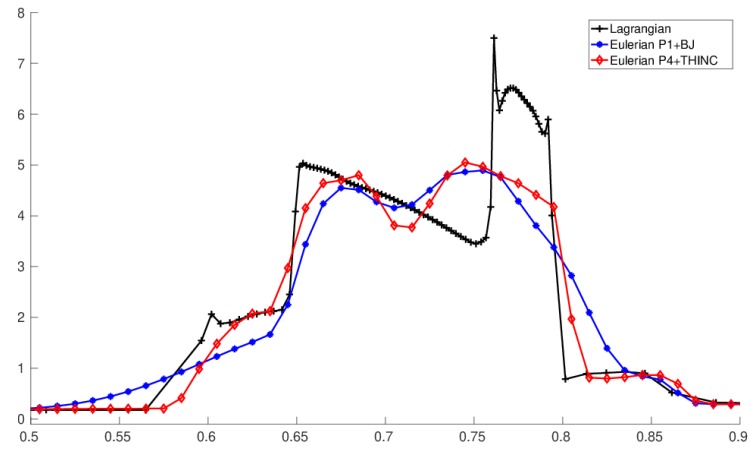

(a) $I=100$, density zoom

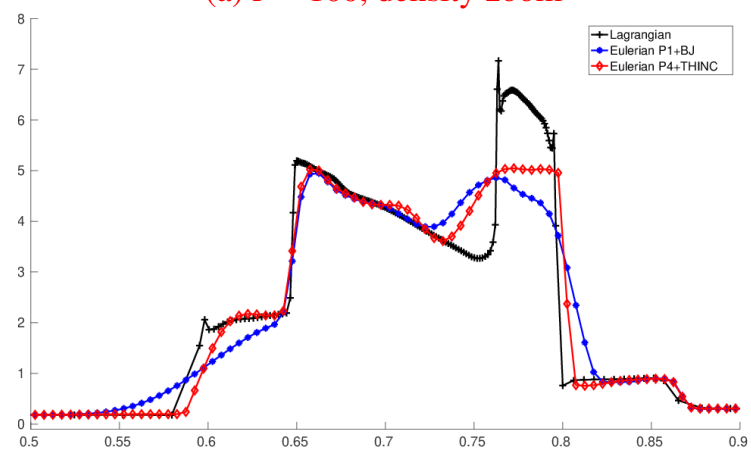

(c) $I=200$, density zoom

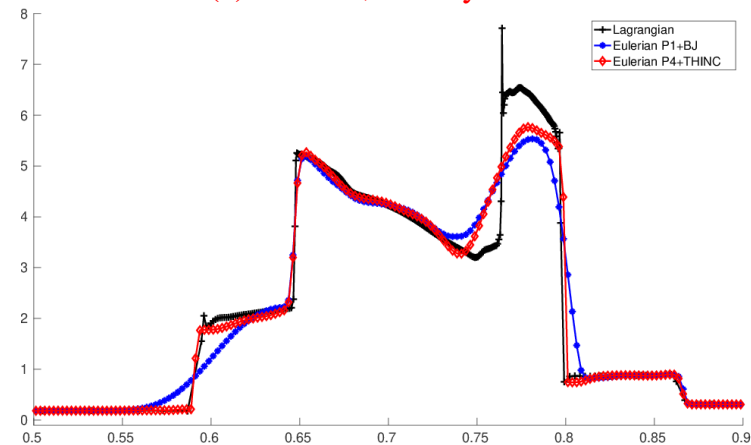

(e) $I=400$, density zoom

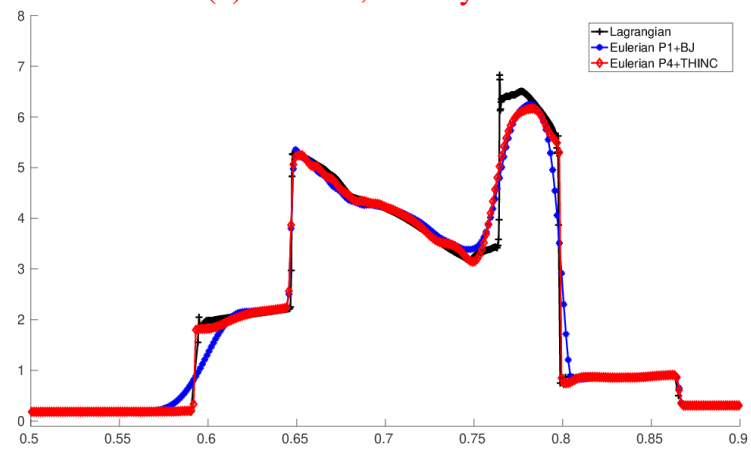

(g) $I=800$, density zoom

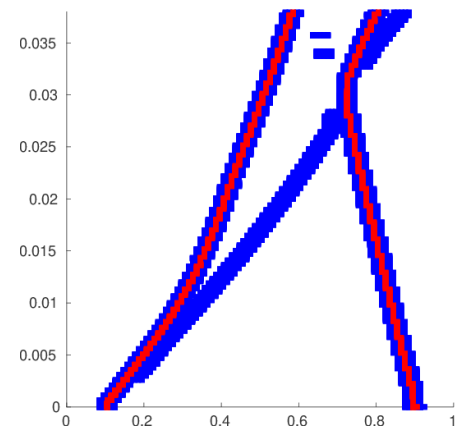

(b) $I=100$, reconstruction type

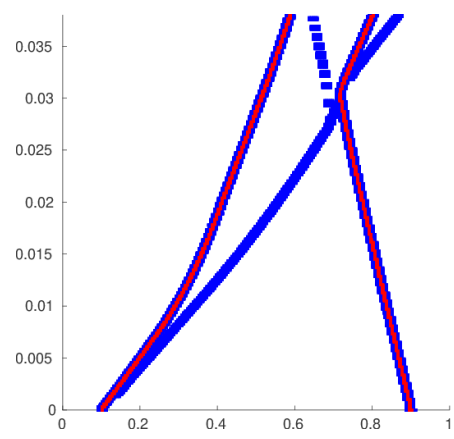

(d) $I=200$, reconstruction type

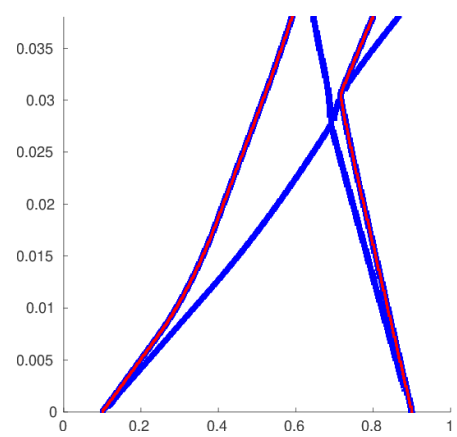

(f) $I=400$, reconstruction type

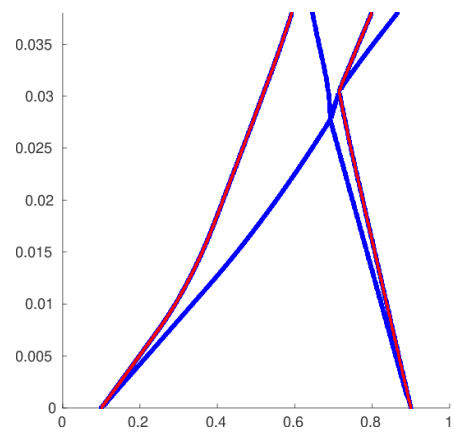

(h) $I=800$, reconstruction type

Figure 16: Woodward-Collela blastwave test case: fluid density shown on 100 (a-b), 200 (c-d), 400 (e-f), and 800 (g-h) cells. Zoom on the central area (left panels) and cell reconstruction types as a function of time (right panels) are shown. 


\section{Conclusions and perspectives}

In this paper, we have presented a 1D proof of concept for the mixed use of polynomial and nonpolynomial THINC reconstructions within a conservative remap procedure of an indirect cell-centered ALE scheme (Lagrange+Rezone+Remap). The use of hyperbolic tangent THINC reconstructions allow us to better represent discontinuous solutions, such as interfaces or contact discontinuity. For safe regular zones we employ quartic polynomial reconstructions resulting in fifth-order accurate remap, while for unsafe zones we use the classical second order accurate remap employing linear reconstruction supplemented with Barth-Jespersen slope limiter. Tracking the discontinuities is therefore mandatory in out approach. Contact discontinuities are moving with the fluid velocity. Therefore, they are initially marked and further tracked with the Lagrangian piece-wise linear approximation of the fluid velocity. In the cells where those markers are present, we perform a THINC reconstruction. Their neighbors are reconstructed with a limited linear polynomial. Concerning shock waves, we rely on a sort of compression switch by stating that if the volume of the Lagrangian cell is enduring a 'large' compression then the situation is not safe and limited linear polynomial must be employed for this cell and its two neighbors. All other cells employ a fifth-order accurate unlimited polynomial reconstruction.

The associated cell-centered ALE scheme therefore employs a second-order accurate cell-centered Lagrangian solver, a simple rezone strategy, and a remapping procedure for which the reconstruction if chosen between $\mathbb{P}_{4}, \mathbb{P}_{1}^{\lim }$ or THINC, depending on a tracking algorithm.

We have then presented the indirect ALE scheme solving the 1D advection equation and the 1D hydrodynamics system of conservation laws. In all these situations, maintaining sharp contact discontinuities is the main goal of our approach.

This remap procedure has been tested on pure reconstruction and static remap situations, and some classical 1D hydrodynamics ones (Sod, Lax, Shu-Osher and Woodward-Collela). The numerical results have been compared to the ones produces by standard piece-wise linear reconstructions supplemented with a slope limiter. The results are genuinely promising. Indeed, it is observed that the THINC reconstructions sharpen the discontinuous profiles without degrading the other regions. For instance, the contact discontinuity is maintained within one cell while at least four cells are usually needed with classical schemes. The $\mathbb{P}_{4}$ reconstructions, on the other hand, allow to maintain good accuracy, and the $\mathbb{P}_{1}^{\text {lim }}$ assures an essentially non-oscillatory behavior. Obviously, our new ALE scheme is more computationally expensive than the classical second-order one but the improvement in terms of accuracy does compensate. Of course, the Lagrangian scheme being only second-order, we can not expect more than a second-order ALE scheme. However it does not imply that the overall accuracy of the numerical results can not be improved, and, we have shown that such a mixed reconstruction approach can genuinely ameliorate the situation. This work was intended to show that the drawbacks brought by the remap stage, such as dissipation of contacts and interfaces, could be truly mitigated by an appropriate choice of reconstruction types. This 1D proof of concept is the first step in this direction.

In the future, we plan to test the full approach in 2D Cartesian geometry for structured and unstructured mesh. Moreover, we plan to improve the tracking algorithm to allow the appearance of contact or interface after some wave interactions. Also extending the tracking algorithm to make it work with the THINC reconstruction to multi-dimension may be a an interesting challenge.

\section{Acknowledgments}

RL would like to thank F. Xiao (Tokyo Tech, Tokyo, Japan) and his students for having introduced him to their THINCking world. MK was supported in parts by the European Regional Development Fund project CZ.02.1.01/0.0/0.0/16_019/0000778, the Czech Science Foundation project 18-20962S, and the Czech Ministry of Education project RVO 68407700. 


\section{In memoriam}

This paper is dedicated to the memory of Dr. Douglas Nelson Woods (*January $11^{\text {th }} 1985-\uparrow$ September

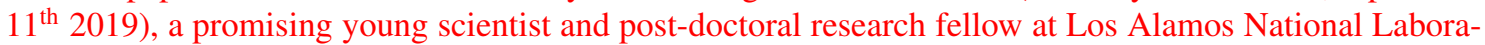
tory. Our thoughts and wishes go to his wife Jessica, to his parents Susan and Tom, to his sister Rebecca and to his brother Chris, whom he left behind.

\section{References}

\section{References}

[1] R. D. Richtmyer, K. W. Morton, Difference Methods for Initial Value Problems (2nd ed.), Wiley, New York, 1967, iSBN 0894647636. 2, 8

[2] H. A. Bethe, R. Christy, R. R. Davis, R. P. Feynman, S. Frankel, E. J. Konopinski, N. Livesay, N. Metropolis, E. Nelson, IBM calculations of implosion hydrodynamics, Tech. Rep. LA-94, Los Alamos National Laboratory (1944). 2

[3] J. V. Neumann, R. D. Richtmyer, A method for the numerical calculation of hydrodynamic shocks, Journal of Applied Physics 21 (3) (1950) 233-237. 2, 6

[4] M. L. Wilkins, Calculation of elastic plastic flow, in: B. Alder, S. Fernbach, M. Rotenberg (Eds.), Methods in Computational Physics, Vol. 3, Academic Press, New York, 1964. 2, 6

[5] D. E. Burton, Advances in the Free-Lagrange Method Including Contributions on Adaptive Gridding and the Smooth Particle Hydrodynamics Method, Vol. 395 of Lecture Notes in Physics, Springer, 1990, Ch. Exact conservation of energy and momentum in staggered-grid hydrodynamics with arbitrary connectivity, pp. 7-19, iSBN 978-3-540-54960-4. 2

[6] E. J. Caramana, D. E. Burton, M. J. Shashkov, P. P. Whalen, The construction of compatible hydrodynamics algorithms utilizing conservation of total energy, Journal of Computational Physics 146 (1) (1998) 227-262. 2, 6

[7] A. L. Bauer, D. E. Burton, E. J. Caramana, R. Loubère, M. J. Shashkov, P. P. Whalen, The internal consistency, stability, and accuracy of the discrete, compatible formulation of Lagrangian hydrodynamics, Journal of Computational Physics 218 (2) (2006) 572-593. 2, 6

[8] R. Loubère, P.-H. Maire, B. Rebourcet, Staggered and colocated finite volume schemes for Lagrangian hydrodynamics, in: R. Abgrall, C.-W. Shu (Eds.), Handbook of Numerical Methods for Hyperbolic Problems Basic and Fundamental Issues, Vol. 17 of Handbook of Numerical Analysis, Elsevier, 2016, Ch. 13, pp. 319-352, iSBN 9780444637895. 2, 6

[9] S. K. Godunov, A. Zabrodine, M. Ivanov, A. Kraiko, G. Prokopov, Résolution numérique des problèmes multidimensionnels de la dynamique des gaz, Mir, 1979. 2, 6, 7

[10] B. Després, C. Mazeran, Lagrangian gas dynamics in two dimensions and Lagrangian systems, Archive for Rational Mechanics and Analysis 178 (3) (2005) 327-372. 2, 6

[11] P.-H. Maire, R. Abgrall, J. Breil, J. Ovadia, A cell-centered Lagrangian scheme for two-dimensional compressible flow problems, SIAM Journal on Scientific Computing 29 (4) (2007) 1781-1824. 2, 6

[12] D. E. Burton, T. C. Carney, N. R. Morgan, S. K. Sambasivan, M. J. Shashkov, A cell-centered Lagrangian Godunov-like method for solid dynamics, Computers \& Fluids 83 (2013) 33-47. 2

[13] J. Cheng, C.-W. Shu, A cell-centered Lagrangian scheme with the preservation of symmetry and conservation properties for compressible fluid flows in two-dimensional cylindrical geometry, Journal of Computational Physics 229 (19) (2010) $7191-$ 7206. 2

[14] J. Qi, B. Tian, J. Li, A high-resolution cell-centered Lagrangian method with a vorticity-based adaptive nodal solver for twodimensional compressible Euler equations, Communications in Computational Physics 24 (2018) 774-790. 2

[15] A. J. Barlow, P. L. Roe, A cell centred Lagrangian Godunov scheme for shock hydrodynamics, Computers \& Fluids 46 (1) (2011) 133-136. 2

[16] G. Carré, S. D. Pino, B. Després, E. Labourasse, A cell-centered Lagrangian hydrodynamics scheme on general unstructured meshes in arbitrary dimension, Journal of Computational Physics 228 (14) (2009) 5160-5183. 2

[17] D. Fridrich, R. Liska, B. Wendroff, Some cell-centered Lagrangian LaxWendroff HLL hybrid schemes, Journal of Computational Physics 326 (2016) 878-892. 2

[18] C. W. Hirt, A. A. Amsden, J. L. Cook, An arbitrary Lagrangian-Eulerian computing method for all flow speeds, Journal of Computational Physics 14 (3) (1974) 227-253. 2

[19] D. J. Benson, Computational methods in Lagrangian and Eulerian hydrocodes, Computer Methods in Applied Mechanics and Engineering 99 (2-3) (1992) 235-394. 2

[20] D. J. Benson, An efficient, accurate, simple ALE method for nonlinear finite element programs, Computer Methods in Applied Mechanics and Engineering 72 (3) (1989) 305-350. 2

[21] P.-H. Maire, J. Breil, S. Galera, A cell-centered arbitrary Lagrangian-Eulerian (ALE) method, International Journal for Numerical Methods in Fluids 56 (8) (2008) 1161-1166. 2

[22] R. Loubère, P.-H. Maire, M. Shashkov, J. Breil, S. Galera, ReALE: A reconnection-based arbitrary-LagrangianEulerian method, Journal of Computational Physics 229 (12) (2010) 4724-4761. 2 
[23] R. Loubère, Contribution to Lagrangian and Arbitrary-Lagrangian-Eulerian numerical schemes, habilitation, University of Toulouse, France (2013).

URL http://loubere.free.fr/Habilitation_Loubere.pdf 2

[24] A. J. Barlow, P.-H. Maire, W. J. Rider, R. N. Rieben, M. J. Shashkov, Arbitrary Lagrangian-Eulerian methods for modeling high-speed compressible multimaterial flows, Journal of Computational Physics 322 (2016) 603-665. 2, 6

[25] J. P. Boris, D. L. Book, Flux-corrected transport. I. SHASTA, a fluid transport algorithm that works, Journal of Computational Physics 11 (1) (1973) 38-69. 2

[26] L. G. Margolin, M. Shashkov, Second-order sign-preserving conservative interpolation (remapping) on general grids, Journal of Computational Physics 184 (1) (2003) 266-298. 2, 3, 5, 14

[27] M. Kucharik, M. Shashkov, B. Wendroff, An efficient linearity-and-bound-preserving remapping method, Journal of Computational Physics 188 (2) (2003) 462-471. 2, 3, 5

[28] J. Grandy, Conservative remapping and region overlays by intersecting arbitrary polyhedra, Journal of Computational Physics 148 (2) (1999) 433-466. 2

[29] R. Loubère, M. Shashkov, A subcell remapping method on staggered polygonal grids for arbitrary-Lagrangian-Eulerian methods, Journal of Computational Physics 209 (1) (2005) 105-138. 2, 5

[30] M. Kucharik, M. Shashkov, Conservative multi-material remap for staggered multi-material arbitrary Lagrangian-Eulerian methods, Journal of Computational Physics 258 (2014) 268-304. 2

[31] B. van Leer, Towards the ultimate conservative difference scheme. II. Monotonicity and conservation combined in a second-order scheme, Journal of Computational Physics 14 (4) (1974) 361-370. 3, 8

[32] B. van Leer, Towards the ultimate conservative difference scheme III. Upstream-centered finite-difference schemes for ideal compressible flow, Journal of Computational Physics 23 (3) (1977) 263-275. 3, 8

[33] B. van Leer, Towards the ultimate conservative difference scheme. V. A second-order sequel to Godunov's method, Journal of Computational Physics 32 (1) (1979) 101-136. 3, 8

[34] G. D. van Albada, B. van Leer, W. W. Roberts, A comparative study of computational methods in cosmic gas dynamics, Astronomy and Astrophysics 108 (1) (1982) 76-84. 3,8

[35] P. K. Sweby, High resolution schemes using flux limiters for hyperbolic conservation laws, SIAM Journal on Numerical Analysis 21 (5) (1984) 995-1011. 3, 8

[36] T. J. Barth, D. C. Jespersen, The design and application of upwind schemes on unstructured meshes, in: AIAA-89-0366, 1989, 27th Aerospace Sciences Meeting, January 9-12, Reno, Nevada. 3, 8, 10

[37] V. Venkatakrishnan, Convergence to steady state solutions of the Euler equations on unstructured grids with limiters, Journal of Computational Physics 118 (1) (1995) 120-130. 3, 8, 13

[38] B. Koren, A robust upwind discretization method for advection, diffusion and source terms, in: C. B. Vreugdenhil, B. Koren (Eds.), Numerical Methods for Advection-Diffusion Problems, Vol. 45 of Notes on Numerical Fluid Mechanics, Vieweg, 1993, pp. 117-138. 3, 8

[39] D. Kuzmin, On the design of general-purpose flux limiters for finite element schemes. I. Scalar convection, Journal of Computational Physics 219 (2) (2006) 513-531. 3, 8

[40] P. L. Roe, Characteristic-based schemes for the Euler equations, Annual Review of Fluid Mechanics 18 (1) (1986) 337-365. 3, 8

[41] L. White, A. Adcroft, A high-order finite volume remapping scheme for nonuniform grids: The piecewise quartic method (PQM), Journal of Computational Physics 227 (15) (2008) 7394-7422. 3, 5

[42] J. Velechovský, R. Liska, M. Shashkov, High-order remapping with piece-wise parabolic reconstruction, Computers \& Fluids 83 (2013) 164-169. 3, 5

[43] G. Blanchard, R. Loubère, High order accurate conservative remapping scheme on polygonal meshes using a posteriori MOOD limiting, Computers \& Fluids 136 (2016) 83-103. 3, 5

[44] F. Xiao, K. Honma, T. Kono, A simple algebraic interface capturing scheme using hyperbolic tangent function, International Journal for Numerical Methods in Fluids 48 (2005) 1023-1040. 3, 9, 11

[45] F. Xiao, S. Ii, C. Chen, Revisit to the THINC scheme: A simple algebraic VOF algorithm, Journal of Computational Physics 230 (19) (2011) 7086-7092. 3, 9, 11

[46] K.-M. Shyue, F. Xiao, An Eulerian interface sharpening algorithm for compressible two-phase flow: The algebraic THINC approach, Journal of Computational Physics 268 (2014) 326-354. 3, 9, 11

[47] Z. Sun, S. Inaba, F. Xiao, Boundary variation diminishing (bvd) reconstruction: A new approach to improve godunov schemes, Journal of Computational Physics 322 (2016) 309-325. 3, 9, 11

[48] X. Deng, S. Inaba, B. Xie, K.-M. Shyue, F. Xiao, High fidelity discontinuity-resolving reconstruction for compressible multiphase flows with moving interfaces, Journal of Computational Physics 371 (2018) 945-966. 3, 9, 11

[49] X. Deng, B. Xie, R. Loubère, Y. Shimizu, F. Xiao, Limiter-free discontinuity-capturing scheme for compressible gas dynamics with reactive fronts, Computers \& Fluids 171 (2018) 1-14. 3, 9, 11

[50] J. K. Dukowicz, J. W. Kodis, Accurate conservative remapping (rezoning) for arbitrary Lagrangian-Eulerian computations, SIAM Journal on Scientific and Statistical Computing 8 (3) (1987) 305-321. 5

[51] L. G. Margolin, M. Shashkov, Remapping, recovery and repair on staggered grid, Computer Methods in Applied Mechanics and Engineering 193 (2004) 4139-4155. 5

[52] M. Kucharik, M. Shashkov, Extension of efficient, swept-integration based conservative remapping method for meshes with changing connectivity, International Journal for Numerical Methods in Fluids 56 (8) (2008) 1359-1365. 5 
[53] E. J. Caramana, M. J. Shashkov, Elimination of artificial grid distortion and hourglass-type motions by means of Lagrangian subzonal masses and pressures, Journal of Computational Physics 142 (2) (1998) 521-561. 6

[54] E. J. Caramana, M. J. Shashkov, P. P. Whalen, Formulations of artificial viscosity for muti-dimensional shock wave computations, Journal of Computational Physics 144 (2) (1998) 70-97. 6

[55] P.-H. Maire, R. Loubère, P. Váchal, Staggered Lagrangian discretization based on cell-centered Riemann solver and associated hydrodynamics scheme, Communications in Computational Physics 10 (4) (2011) 940-978. 6

[56] P.-H. Maire, A high-order cell-centered Lagrangian scheme for two-dimensional compressible fluid flow on unstructured meshes, Journal of Computational Physics 228 (7) (2009) 2391-2425. 6, 8, 9

[57] P.-H. Maire, A unified sub-cell force-based discretization for cellcentered Lagrangian hydrodynamics on polygonal grids, International Journal for Numerical Methods in Fluids 65 (11-12) (2011) 1281-1294. 6

[58] P.-H. Maire, Contribution to the numerical modeling of inertial confinement fusion, habilitation, University of Bordeaux, France (2011). 6, 9

[59] P.-H. Maire, J. Breil, A secondorder cellcentered Lagrangian scheme for twodimensional compressible flow problems, International Journal for Numerical Methods in Fluids 56 (8) (2008) 1417-1423. 6

[60] P.-H. Maire, A high-order cell-centered Lagrangian scheme for compressible fluid flows in two-dimensional cylindrical geometry, Journal of Computational Physics 228 (18) (2009) 6882-6915. 8

[61] B. van Leer, A historical oversight: Vladimir P. Kolgan and his high-resolution scheme, Journal of Computational Physics 230 (7) (2011) 2378-2383. 8

[62] V. P. Kolgan, Application of the principle of minimizing the derivative to the construction of finite-difference schemes for computing discontinuous solutions of gas dynamics, Journal of Computational Physics 230 (7) (2011) 2384-2390. 8

[63] J. K. Dukowicz, A general, non-iterative Riemann solver for Godunov's method, Journal of Computational Physics 61 (1) (1985) 119-137. 9, 13

[64] M. Ben-Artzi, J. Falkovitz, A second-order Godunov-type scheme for compressible fluid dynamics, Journal of Computational Physics 55 (1) (1984) 1-32. 9, 13

[65] M. Ben-Artzi, J. Falkovitz, Generalized Riemann Problems in Computational Fluid Dynamics, Cambridge University Press, 2003, iSBN 9780511546785. 9, 13

[66] M. Ben-Artzi, J. Falkovitz, An upwind second-order scheme for compressible duct flows, SIAM Journal on Scientific and Statistical Computing 7 (3) (1986) 744-768. 9, 13

[67] M. Dumbser, M. Käser, Arbitrary high order non-oscillatory finite volume schemes on unstructured meshes for linear hyperbolic systems, Journal of Computational Physics 221 (2) (2007) 693-723. 9

[68] M. Dumbser, M. Käser, V. A. Titarev, E. F. Toro, Quadrature-free non-oscillatory finite volume schemes on unstructured meshes for nonlinear hyperbolic systems, Journal of Computational Physics 226 (1) (2007) 204-243. 9

[69] M. Dumbser, D. S. Balsara, E. F. Toro, C.-D. Munz, A unified framework for the construction of one-step finite volume and discontinuous Galerkin schemes on unstructured meshes, Journal of Computational Physics 227 (18) (2008) 8209-8253. 9

[70] W. Boscheri, M. Dumbser, R. Loubère, P.-H. Maire, A second-order cell-centered Lagrangian ADER-MOOD finite volume scheme on multidimensional unstructured meshes for hydrodynamics, Journal of Computational Physics 358 (2018) 103-129. 9

[71] S. Diot, S. Clain, R. Loubère, Improved detection criteria for the multi-dimensional optimal order detection (MOOD) on unstructured meshes with very high-order polynomials, Computers \& Fluids 64 (2012) 43-63. 10

[72] C. F. Ollivier-Gooch, High-order ENO schemes for unstructured meshes based on least-squares reconstruction, in: AIAA Meeting Paper, 1997, 35th Aerospace Sciences Meeting and Exhibit, Reno, USA, 1997. 10

[73] M. Yang, Z. J. Wang, A parameter-free generalized moment limiter for high-order methods on unstructured grids, Advances in Applied Mathematics and Mechanics 1 (2009) 451-480. 15

[74] F. Vilar, A high-order Discontinuous Galerkin discretization for solving two-dimensional Lagrangian hydrodynamics, Ph.D. thesis, Universite Bordeaux I (2012). 15

[75] G. A. Sod, A survey of several finite difference methods for systems of nonlinear hyperbolic conservation laws, Journal of Computational Physics 27 (1) (1978) 1-31. 19

[76] E. F. Toro, Riemann Solvers and Numerical Methods for Fluid Dynamics, Springer Verlag, Berlin, Heidelberg, 1997, iSBN 3-540-61676-4. 19, 20, 21

[77] P. Woodward, P. Colella, The numerical simulation of two-dimensional fluid flow with strong shocks, Journal of Computational Physics 54 (1) (1984) 115-173. 19, 22

[78] C.-W. Shu, S. Osher, Efficient implementation of essentially non-oscillatory shock-capturing schemes, II, Journal of Computational Physics 83 (1) (1989) 32-78. 19, 21 